\title{
Spectropolarimetry of magnetic stars
}

\section{Longitudinal field, crossover and quadratic field: New measurements ${ }^{\star}$}

\author{
G. Mathys ${ }^{1}$ and S. Hubrig ${ }^{2}$ \\ 1 European Southern Observatory, Casilla 19001, Santiago 19, Chile \\ 2 University of Potsdam, Am Neuen Palais 10, D-14469 Potsdam, Germany
}

Received November 20, accepted December 4, 1996

\begin{abstract}
New determinations of the mean longitudinal magnetic field, of the crossover, and of the mean quadratic magnetic field of Ap stars are presented. They are based on spectra recorded simultaneously in both circular polarizations at ESO with the CASPEC spectrograph fed by the $3.6 \mathrm{~m}$ telescope. This paper discusses 95 observations of 44 stars. A major result of this study is the discovery that HD 137509 has a predominantly quadrupolar magnetic field, a strucuture previously found in only a couple of stars. Improvement or revision of the determination of the rotation period has been achieved for 3 stars. The stars studied in this work include 14 rapidly oscillating Ap stars (for 6 of which no previous attempt to detect a magnetic field had ever been made) and 21 Ap stars with spectral lines resolved into their magnetically split components when observed at high enough dispersion in unpolarized light (for 9 of these stars, no determination of the longitudinal field had been performed before).

The observations discussed in this paper have been performed between 1989 and 1994, a period during which CASPEC and its Zeeman analyzer have progressively undergone various configuration changes. The results reported here demonstrate that the polarimetric performance of the instrument has remained unaltered through these modifications. Thanks to the latter, the achieved resolving power was increased, which resulted in improved magnetic measurement accuracies.
\end{abstract}

Key words: stars: chemically peculiar — stars: magnetic fields — stars: HD 137509

\section{Introduction}

The papers of this series are devoted to the diagnosis of the magnetic field of Ap stars from the consideration of the polarization of their spectral lines. Particular attention is

Send offprint requests to: G. Mathys

* Based on observations collected at the European Southern Observatory (La Silla, Chile; ESO programmes Nos. 47.7-045 and 49.7-029). paid to the information contents of the profiles of the polarized lines, an aspect whose study has become possible thanks to the use of modern linear detectors such as CCDs. The first paper of the series (Mathys \& Stenflo 1986; hereafter Paper I) introduced the kind of analysis techniques that was contemplated for this project. Paper II (Mathys 1991) presented measurements of the mean longitudinal magnetic field of a sample of Ap stars. The uncertainties affecting the diagnosis of stellar magnetic fields through the methods applied in this series of papers were discussed in general terms in Paper III (Mathys 1994). The application of the results of this discussion to the determination of the longitudinal field led to the revision of the values of the latter that had been derived in Paper II. The first ever quantitative measurements of the crossover were reported in Paper IV (Mathys 1995a). Paper V (Mathys 1995b) was devoted to the mean quadratic magnetic field.

The circularly polarized spectra analyzed in Papers I to $\mathrm{V}$ had all been recorded between 1985 and 1988 with the same configuration of CASPEC (the ESO Cassegrain Echelle Spectrograph, attached to the ESO $3.6 \mathrm{~m}$ telescope) and of its Zeeman analyzer. This configuration has been described in detail in Paper I. Between 1989 and 1994, CASPEC and its Zeeman analyzer underwent a number of modifications, to bring them to their current configuration, which has been stable since early 1995 . We present here the magnetic field determinations performed using observational data obtained during the 1989-1994 transition period (in part as check of the proper operation of the instrument after its various modifications). The magnetic fields have been diagnosed through application of the methods described in Papers III to V. The present data complement the results reported in those papers. In particular, new observations have been obtained for several of the stars studied therein, improving the phase coverage of their variations and the definition of their magnetic curves. On the other hand, we also present magnetic data for a number of stars that we had never studied before. These data are still very incomplete, but they are 
often the first measurements based on spectropolarimetry of the magnetic fields of the considered stars, which should confer them enough interest to warrant publication.

In the next section, we describe the observations and their reduction. Section 3 summarizes the methods of analysis. The resulting magnetic field measurements are presented and discussed, star by star, in Sect. 4 . The main results of this study and the conclusions that can be drawn from them are summarized in Sect. 5.

\section{Observations and reduction}

The observations reported here were performed with the ESO $3.6 \mathrm{~m}$ telescope and its Cassegrain Echelle Spectrograph (CASPEC) on the following dates: May 2125, 1991; June 7-9, 1992; August 10-13, 1992; February 78, 1993; and July 24-27, 1993. The Zeeman analyzer built into CASPEC was inserted in the light path to separate incoming light of right and left circular polarization (RCP and LCP, resp.), and the spectra corresponding to both polarizations were recorded simultaneously, interleaved as described in Paper I.

The configuration of the Zeeman analyzer used in 1991 and 1992 is identical to the one that had been employed between 1985 and 1988 to record the spectra from which the magnetic results presented in Papers I to V had been derived. A detailed description of this configuration has been given in Paper I. It appears useful to summarize it here for understandability of the differences introduced in the instrumental setup after 1992. Namely, the analyzer was originally a self-contained unit mounted behind the entrance slit of the spectrograph on the wheel also bearing the "rear slit viewer" used to center the target in the slit at acquisition time. This wheel is remotely controlled via the instrument computer, so that the analyzer can be called into the light beam at exposure definition time through the observation interface. Within the analyzer, the light beam passes consecutively through:

- a first set of lenses, to convert the diverging beam into a parallel beam;

- an achromatic quarter-wave plate, to convert incoming opposite circular polarizations into mutually orthogonal linear polarizations;

- a Wollaston prism, to separate these two orthogonal linear polarizations;

- a second set of lenses, to bring back the two emergent light beams to the original focal ratio of the incoming beam. In principle, this design guarantees that the focus of CASPEC is unaffected by the presence of the Zeeman analyzer in the light beam. In practice, a small focus offset is introduced by the Zeeman analyzer, which is easily accounted for when defining an exposure by introducing the appropriate correction of the (remotely controlled) collimator position through the observation software.
The two light beams coming out of the Zeeman analyzer have polarization directions resp. parallel and orthogonal to the grooves of the echelle grating, so that they are reflected by the latter with different efficiencies. This results in differences in the continuum level between the spectra corresponding to incoming light of RCP and LCP. This difference is compensated for at reduction time by renormalizing the spectra of both polarizations to the same continuum level of 1 . This is acceptable because we are interested only in the polarization of spectral lines in objects whose continuum has no significant circular polarization.

From 1993, the Zeeman analyzer of CASPEC has been modified as followed (the February 1993 observations reported here were the first test of this modified configuration):

- within the self-contained unit borne by the "rear slit viewer wheel", the quarter-wave plate and the Wollaston prism have been interchanged;

- an additional achromatic quarter-wave plate has been installed in a filter wheel located between the spectrograph entrance slit and the "rear slit viewer wheel", whose original purpose is to hold interferential filters used to select a single echelle order in view of recording long-slit (not cross-dispersed) spectra of extended objects. As the other functions of CASPEC, this "longslit filter wheel" is remotely controlled through the observation software.

In this new configuration, the stellar light first goes through the quarter-wave plate located in the "long-slit filter wheel". As a result, incoming circular polarizations are converted to mutually orthogonal linear polarizations. These linear polarizations are separated by the Wollaston prism of the unit borne by the "rear slit viewer wheel". The two resulting light beams are converted back to circular polarization by the quarter-wave plate following the Wollaston prism within the analyzer unit. This allows them to be transmitted with the same efficiency by the subsequent elements of the optical train.

There are a number of additional differences of instrumental setup between the various observing runs reported in this paper, and with respect to the homogeneous set of observations discussed in Papers I to V. They will now be described.

In May 1991 and in June 1992, we used the same camera ("short camera", $f / 1.6)$ and echelle grating (52 lines $/ \mathrm{mm}$ ) as between 1985 and 1988. But the CCD RCA \#3 $\left(512 \times 320\right.$ pixels of $\left.30 \times 30 \mu \mathrm{m}^{2}\right)$ that we had previously employed was replaced by the CCD Tektronix \#16 $\left(512 \times 512\right.$ pixels of $\left.27 \times 27 \mu \mathrm{m}^{2}\right)$. As a result, a slightly higher spectral resolution was achieved $\left(\lambda / \Delta \lambda \approx 1.810^{4}\right)$, and a significantly longer spectral range was recorded overall (from $5400 \AA$ to $6800 \AA$ ), although at the expense of somewhat larger wavelength gaps between consecutive echelle orders. 
In August 1992, the "long camera" ( $f / 3$, Pasquini 1993) of CASPEC was for the first time used in combination with the Zeeman analyzer (still in its old configuration). The larger interorder separation then achieved on the CCD allowed the use of the 31.6 lines $/ \mathrm{mm}$ echelle grating without overlapping of opposite polarizations of consecutive orders (see Paper I). The efficiency of this grating in the spectral region of interest is higher than that of the 52 lines/mm grating, which compensates to some extent for the increased slit losses (for full exploitation of the resolution achievable with the long camera, $\lambda / \Delta \lambda \approx 3.510^{4}$, the slit width was set to $1^{\prime \prime} 05$, compared to 2 '. 1 with the short camera). Also, all other things being equal, the use of the 31.6 lines $/ \mathrm{mm}$ grating would reduce the interorder gaps in the wavelength coverage, compared to the 52 lines $/ \mathrm{mm}$. However, with the small size of CCD \#16 (which was mounted for the considered run), the increase in the geometrical scale between the short and the long camera more than cancels this advantage, since a large fraction of the whole cross-dispersed echelle pattern falls outside the detector boundaries. As a result, the spectra range only from $5710 \AA$ to $6450 \AA$, with gaps of $17 \AA$ to $27 \AA$ between consecutive orders.

The first tests of the modified configuration of the Zeeman analyzer in February 1993 were carried out with essentially the same setup as used in May 1991 and June 1992: 52 lines/mm echelle grating and short camera. This time, the CCD was \#32, a Tektronix chip having the same format as \#16 but a higher efficiency in the blue-violet part of the spectrum: that is, this detector was essentially equivalent to \#16 for our purpose.

CCD \#32 was also used for the observations of July 1993, together with the configuration of the Zeeman analyzer introduced in Februray 1993, the 31.6 lines/mm echelle grating, and the long camera of CASPEC.

This latter configuration is closest to the present standard setup of CASPEC for observations with the Zeeman analyzer. The only difference is that CCD \#32 has been replaced by \#37, a Tektronix of $1024 \times 1024$ pixels of $24 \times 24 \mu \mathrm{m}^{2}$. With this setup, spectra can now be recorded at a resolving power $\lambda / \Delta \lambda \approx 3.910^{4}$ over the range $5600 \AA-6800 \AA$, without gaps in the wavelength coverage. This configuration is mentioned here only for completeness of the record. Using it, we are currently regularly obtaining observations for a new programme, the results of which will be presented in a future paper.

The various configurations of CASPEC and of its Zeeman analyzer that we have employed for studies of stellar magnetic fields are summarized in Table 1 . The dates at which each configuration was used are given in Col. 1 . The two configurations of the Zeeman analyzer (ZA) itself, which have been described above, are called "old" and "new" in Col. 2. The echelle grating is referred to in Col. 3 by its number of lines per millimeter. Since 1991, CASPEC users have been offered the choice between two cross-dispersers (X-disp.), a "blue" one and a "red" one, which are optimized to give a better efficiency resp. at blue or at red wavelengths. Although the red cross-disperser should be slightly more efficient than the blue one in the spectral range covered by our observations, it is unsuitable for our purpose: when using it, the echelle orders are too packed, and opposite polarisations of consecutive orders would overlap when the Zeeman analyzer is inserted in the light beam. Accordingly, we have always used the blue cross-disperser, as indicated in Col. 4 of Table 1. Columns 5 and 6 of this table give the camera used (short or long), and the CCD (referred to by its internal ESO number).

Table 1. Configurations of CASPEC and of its Zeeman analyzer used to study stellar magnetic fields

\begin{tabular}{llllll}
\hline Dates & ZA & $\begin{array}{l}\text { echelle } \\
(\text { lines } / \mathrm{mm})\end{array}$ & X-disp. & camera & CCD \\
\hline 1985-1988 & old & 52 & blue & short & $\# 3$ \\
May 1991 & old & 52 & blue & short & $\# 16$ \\
June 1992 & old & 52 & blue & short & $\# 16$ \\
August 1992 & old & 31.6 & blue & long & $\# 16$ \\
February 1993 & new & 52 & blue & short & $\# 32$ \\
July 1993 & new & 31.6 & blue & long & $\# 32$ \\
since 1995 & new & 31.6 & blue & long & $\# 37$ \\
\hline
\end{tabular}

The data reduction steps have been described in Paper II. The procedure applied to the present observations differed from the one used for the 1985-1988 data only in two (minor) aspects:

- while a specific FORTRAN code had been written for the reduction of our former spectra, here we used MIDAS procedures built from existing MIDAS commands. This simplification (from the point of view of the user) was made possible by the use of more powerful computers (Unix Sun workstations instead of a VMS Vax 11/750) and by the increased versatility and robustness of the MIDAS echelle package, which permitted us to use standard commands in spite of the non-standard format of the interleaved right and left circularly polarized spectra;

- we gave up using an automatic procedure for normalization of the spectra to the continuum, which we did not always find fully satisfactory. Instead, a polynomial was fitted to the high points of each order, defined interactively by cursor entry.

The reduced spectra have signal-to-noise ratios ranging from 100 to 300 (most of them between 150 and 200). The rms deviation of the lines of the ThAr arc spectra used for wavelength calibration with respect to the fitted dispersion law is of the order of 3 to $3.5 \mathrm{~m} \AA$ with the short camera, and of 1.5 to $1.8 \mathrm{~m} \AA$ with the long camera.

The stars for which magnetic field measurements are reported here are listed in Table 2 . The presentation is 
Table 2. Programme stars

\begin{tabular}{|c|c|c|c|c|c|c|c|c|}
\hline HD & Other id. & $V$ & Sp. type & $P$ & Ref. & $\mathrm{HJD}_{0}$ & Phase origin & Ref. \\
\hline 2453 & $\mathrm{BD}+31^{\circ} 59$ & 6.9 & $\mathrm{~A} 1 \mathrm{p} \mathrm{SrEuCr}$ & $521 \mathrm{~d}$ & 1 & 2442288.000 & $\left\langle H_{z}\right\rangle \min$. & 17 \\
\hline 5737 & $\alpha \mathrm{Scl}$ & 4.3 & B6p He weak & $21^{\mathrm{d}} .652$ & 2 & 2447542.000 & min. brightness in $u$ & 2 \\
\hline 19918 & $\mathrm{CPD}-82^{\circ} 54$ & 9.4 & A5p $\mathrm{SrEuCr}$ & & & & & \\
\hline 22920 & HR 1121 & 5.5 & $\mathrm{~B} 8 \mathrm{p} \mathrm{Si}$ & 3.96 or $1.33 ?$ & 4 & & & \\
\hline 24712 & HR 1217 & 6.0 & A9p $\mathrm{SrEuCr}$ & 12.4610 & 5 & 2440577.230 & $\left\langle H_{\mathrm{z}}\right\rangle$ pos. extr. & 18 \\
\hline 37058 & $\mathrm{BD}-4^{\circ} 1187$ & 7.3 & B3p He weak Sr & 14.612 & 6 & & & \\
\hline 50169 & $\mathrm{BD}-1^{\circ} 1414$ & 9.0 & A3p $\mathrm{SrCrEu}$ & $\gg 4 \mathrm{y}$ & 1 & & & \\
\hline 55719 & HR 2727 & 5.3 & A3p $\mathrm{SrCrEu}$ & $847 \mathrm{~d}$ or $775 \mathrm{~d} ?$ & 1 & & & \\
\hline 70331 & $\mathrm{CoD}-47^{\circ} 3803$ & 8.9 & $\mathrm{~B} 8 \mathrm{p} \mathrm{Si}$ & 3.03 or $3.65 ?$ & 1 & & & \\
\hline 81009 & HR 3724 & 7.2 & A3p CrSrSi & 33.96 & 7 & 2444486.000 & $\langle H\rangle \min$. & 1 \\
\hline 83368 & HR 3831 & 6.3 & $\mathrm{~A} 8 \mathrm{p} \mathrm{SrCrEu}$ & 2.851982 & 8 & 2444576.169 & $\left\langle H_{\mathrm{z}}\right\rangle$ neg. extr. & 8 \\
\hline 116458 & HR 5049 & 5.7 & A0p SiEuCr & 147.9 & 9 & 2447600.000 & arbitrary & 9 \\
\hline 119419 & HR 5158 & 6.5 & A0p SiCrEu & 2. 60090 & 10 & 2448627.000 & $\left\langle H_{z}\right\rangle$ neg. extr. & 10 \\
\hline 125248 & CS Vir & 5.9 & A1p EuCr & 9.2954 & 11 & 2430143.070 & $\left\langle H_{\mathrm{z}}\right\rangle$ pos. extr. & 11 \\
\hline 126515 & $\mathrm{BD}+1^{\circ} 3927$ & 7.1 & $\mathrm{~A} 2 \mathrm{p} \mathrm{CrSr}$ & 129.95 & 1 & 2437015.000 & $\langle H\rangle \max$ & 19 \\
\hline 128898 & $\alpha \operatorname{Cir}$ & 3.2 & A9p SrEu & 4. 4790 & 12 & 2449111.686 & max. puls. amplitude & 12 \\
\hline 134214 & $\mathrm{BD}-13^{\circ} 4081$ & 7.5 & F2p SrEuCr & 4. $1456 ?$ & 1 & & & \\
\hline 137509 & $\mathrm{CPD}-70^{\circ} 2069$ & 6.9 & B8p SiCrFe & 4.4916 & 13 & 2448391.000 & $\left\langle H_{z}\right\rangle$ neg. extr. & 13 \\
\hline 137909 & $\beta \mathrm{CrB}$ & 3.9 & A9p SrEuCr & 18.4868 & 14 & 2434204.700 & $\left\langle H_{\mathrm{z}}\right\rangle$ pos. extr. & 14 \\
\hline 137949 & $33 \mathrm{Lib}$ & 6.7 & F0p SrEuCr & $\gtrsim 75 \mathrm{y} ?$ & 1 & & & \\
\hline 144897 & $\mathrm{CoD}-40^{\circ} 10236$ & 8.6 & B8p EuCr & $48^{\mathrm{d}} 43$ & 1 & 2449133.700 & $\langle H\rangle \min$. & 1 \\
\hline 147010 & $\mathrm{BD}-19^{\circ} 4359$ & 7.4 & B9p $\mathrm{SiCrSr}$ & 3.920676 & 15 & 2446894.515 & max. brightness in $[U]$ & 20 \\
\hline 188041 & HR 7575 & 5.6 & A6p $\mathrm{SrCrEu}$ & 223.9 & 9 & 2434323.000 & $\left\langle H_{z}\right\rangle \min$ & 24 \\
\hline 193756 & $\mathrm{CPD}-52^{\circ} 11681$ & 9.2 & A9p $\mathrm{SrCrEu}$ & & & & & \\
\hline 335238 & $\mathrm{BD}+29^{\circ} 4202$ & 9.2 & $\mathrm{~A} 1 \mathrm{p} \mathrm{CrEu}$ & $44^{\mathrm{d}} 0 ?$ & 1 & & & \\
\hline 201601 & $\gamma \mathrm{Equ}$ & 4.7 & A9p SrEu & $\gtrsim 70 \mathrm{y}$ & 16 & & & \\
\hline 203932 & $\mathrm{CoD}-30^{\circ} 18600$ & 8.8 & A5p SrEu & & & & & \\
\hline 216018 & $\mathrm{BD}-12^{\circ} 6357$ & 7.9 & $\mathrm{~A} 7 \mathrm{p} \mathrm{SrCrEu}$ & $\gg 3 \mathrm{y}$ & 1 & & & \\
\hline 217522 & $\mathrm{CoD}-45^{\circ} 14901$ & 7.5 & A5p $\mathrm{SrEuCr}$ & & & & & \\
\hline 218495 & $\mathrm{CPD}-64^{\circ} 4322$ & 9.4 & $\mathrm{~A} 2 \mathrm{p} \mathrm{EuSr}$ & & & & & \\
\hline
\end{tabular}

References: 1: Mathys et al. (1996) (MHLLM); 2: Manfroid \& Renson (1994); 3: Kurtz et al. (1996); 4: North (1990) (unpublished; cited by Catalano et al. 1991); 5: Mathys (1991) (Paper II); 6: Pedersen (1979); 7: Waelkens (1985); 8: Kurtz et al. (1992); 9: Hensberge (1993); 10: this paper; 11: Babcock (1960); 12: Kurtz et al. (1994); 13: Mathys \& Lanz (1996); 14: Kurtz (1989); 15: Catalano \& Leone (1993); 16: Leroy et al. (1994); 17: Wolff (1975); 18: Kurtz \& Marang (1987); 19: Preston (1970); 20: Lanz \& Mathys (1991); 21: Babcock (1958); 22: Bohlender et al. (1987); 23: Landstreet (unpublished; cited by Mathys 1991); 24: Wolff (1969). 
identical to Table 1 of Paper II (or Table 2 of Paper III): the HD (or HDE) number and another identifier in Cols. 1 and 2, the $V$ magnitude and the spectral type (both from the catalog of Renson et al. 1991) in Cols. 3 and 4, the adopted value of the rotation period $P$ and the reference from which it is retrieved in Cols. 5 and 6 , the heliocentric Julian date taken as the origin of the phases, the particular property (e.g. a magnetic field extremum) from which this origin is defined, and the corresponding reference in Cols. 7 to 9 .

\section{Magnetic field diagnosis}

Stellar magnetic fields are diagnosed from the observational material described in the previous section through application of the moment technique (Mathys 1988), in essentially the same manner as in Papers III to V.

The following moments of the magnetic field have been determined in the present study:

- the mean longitudinal magnetic field $\left\langle H_{\mathrm{z}}\right\rangle$,

- the crossover, that is, the product of the projected equatorial velocity $v \sin i$ and of the mean asymmetry of the longitudinal magnetic field $\left\langle x H_{z}\right\rangle$,

- and the mean quadratic magnetic field $\left(\left\langle H^{2}\right\rangle+\right.$ $\left.\left\langle H_{\mathrm{z}}^{2}\right\rangle\right)^{1 / 2}$.

For the sake of clarity, let us briefly recall the meaning of each of these quantities, and how they are derived. The reader is referred to Mathys $(1988,1989)$ and to Papers II to $\mathrm{V}$ for a discussion of the approximations underlying these derivations.

The mean longitudinal magnetic field (in short, the longitudinal field) is the average over the stellar hemisphere visible at the time of observation of the component of the magnetic field parallel to the line of sight, weighted by the local emergent spectral line intensity. It is diagnosed from the first order moment of the line profiles in the Stokes parameter $V$ (characterizing the circular polarization), that is, from the wavelength shift of the spectral lines between RCP and LCP. The latter can be written under the form:

$\lambda_{\mathrm{R}}-\lambda_{\mathrm{L}}=2 \bar{g} \Delta \lambda_{\mathrm{Z}}\left\langle H_{\mathrm{Z}}\right\rangle$.

$\lambda_{\mathrm{R}}\left(\lambda_{\mathrm{L}}\right)$ is the wavelength of the centre of gravity of the line as recorded in RCP (LCP). $\Delta \lambda_{\mathrm{Z}}=k \lambda_{0}^{2}$ is the Lorentz unit $\left(\lambda_{0}\right.$ is the laboratory wavelength of the line and $\left.k=4.6710^{-13} \AA^{-1} \mathrm{G}^{-1}\right) \cdot \bar{g}$ is the effective Landé factor of the considered transition, which characterizes the displacement of the centre of gravity of its $\sigma_{+}$components with respect to the line centre.

In practice, the wavelength shifts $\left(\lambda_{\mathrm{R}}-\lambda_{\mathrm{L}}\right)$ are measured for a sample of spectral lines. $\left\langle H_{z}\right\rangle$ is determined by performing a linear regression of these measurements as a function of $2 \bar{g} \Delta \lambda_{\mathrm{Z}}$, applying the least-squares method. This linear regression is forced through the origin and weighted by the uncertainties of the measurements of
$\lambda_{\mathrm{R}}-\lambda_{\mathrm{L}}$. The evaluation of the latter is described in detail in Paper III. The standard error $\sigma\left(\left\langle H_{z}\right\rangle\right)$, obtained from the least-squares analysis, is used as an estimate of the uncertainty of the derived longitudinal field.

The mean asymmetry of the longitudinal magnetic field $\left\langle x H_{z}\right\rangle$ (more briefly, the asymmetry of the longitudinal field) is the first-order moment about the plane defined by the line of sight and the stellar rotation axis of the component of the magnetic vector parallel to the line of sight. This moment is computed over the visible stellar hemisphere and weighted by the local emergent spectral line intensity. The quantity which is directly obtained from the observations is the product of the asymmetry of the longitudinal field and of the projected equatorial velocity $v \sin i$. We call this product the crossover. It is derived from the second-order moment about the line centre of the line profile in the Stokes $V$ parameter, $R_{V}^{(2)}\left(\lambda_{0}\right)$. This moment characterizes the difference of the widths of the spectral lines between RCP and LCP (for more about the intuitive interpretation of the low-order moments of the line profiles, see Mathys 1993). The moment $R_{V}^{(2)}\left(\lambda_{0}\right)$ can be expressed as:

$R_{V}^{(2)}\left(\lambda_{0}\right)=2 \bar{g} \Delta \lambda_{\mathrm{Z}} \Delta \lambda_{\mathrm{R}}\left\langle x H_{\mathrm{Z}}\right\rangle$,

where $\Delta \lambda_{\mathrm{R}}=\lambda_{0}(v / c) \sin i$ (as usual, $v$ is the stellar equatorial velocity and $i$ the angle between the rotation axis and the line of sight).

In practice, the crossover is derived like the longitudinal field, by performing a weighted regression analysis over a set of measurements of $R_{V}^{(2)}\left(\lambda_{0}\right)$ for a selected sample of spectral lines; this regression is forced through the origin (for details, see Paper IV).

The mean quadratic magnetic field (or quadratic field) is the square root of the mean square magnetic field. The latter is the sum of two terms:

- the mean square magnetic field modulus $\left\langle H^{2}\right\rangle$, that is, the average over the visible stellar hemisphere of the square of the modulus of the magnetic vector, weighted by the local emergent line intensity;

- the mean square longitudinal magnetic field $\left\langle H_{\mathrm{z}}^{2}\right\rangle$, that is, the average over the visible stellar hemisphere of the square of the component of the magnetic vector parallel to the line of sight, weighted by the local emergent line intensity.

The quadratic field is at least equal and generally greater than the mean magnetic field modulus $\langle H\rangle^{1}$. Indeed, even in the (hypothetical) case of zero contribution of the longitudinal field term $\left\langle H_{\mathrm{z}}^{2}\right\rangle$, the mean quadratic field modulus

1 The mean magnetic field modulus $\langle H\rangle$ is the average over the visible stellar hemisphere of the modulus of the magnetic vector, weighted by the local emergent spectral line intensity. It is diagnosed from the relative wavelength shift of line components split by the magnetic field, as observed in unpolarized light. 
Table 3. Mean longitudinal magnetic field, crossover, and mean quadratic magnetic field

\begin{tabular}{|c|c|c|c|c|c|c|c|c|c|}
\hline $\mathrm{HD}$ & $\begin{array}{l}\text { HJD } \\
(2440000+)\end{array}$ & Phase & $\begin{array}{l}\text { Long. } \\
\text { field } \\
\text { (G) }\end{array}$ & $\sigma_{\mathrm{z}}$ & $\begin{array}{l}\text { Cross- } \\
\text { over } \\
\left(\mathrm{km} \mathrm{s}^{-1}\right.\end{array}$ & $\begin{array}{r}\sigma_{\mathrm{x}} \\
\left.{ }^{1} \mathrm{G}\right)\end{array}$ & $\begin{array}{l}\text { Quad. } \\
\text { field } \\
\text { (G) }\end{array}$ & $\sigma_{\mathrm{q}}$ & \\
\hline 2453 & 9193.866 & 0.255 & -464 & 70 & -1 & 386 & 2445 & 1818 & \\
\hline 5737 & 8847.867 & 312 & -322 & 140 & 2342 & 1521 & 3903 & 2218 & \\
\hline 6532 & 8846.839 & 399 & -517 & & -1870 & 3838 & 21944 & & \\
\hline 19918 & 8845.875 & & -848 & & 2039 & 1390 & 1704 & 7126 & \\
\hline 22920 & 8847.873 & & 351 & & 1740 & 2760 & & & \\
\hline 24712 & & $\begin{array}{l}0.566 \\
0.642 \\
0.720 \\
0.494 \\
0.568\end{array}$ & $\begin{array}{r}129 \\
331 \\
560 \\
19 \\
100\end{array}$ & $\begin{array}{r}75 \\
98 \\
140 \\
176 \\
122\end{array}$ & $\begin{array}{r}-414 \\
-1079 \\
-1032 \\
-2104 \\
-1192\end{array}$ & $\begin{array}{l}785 \\
737 \\
852 \\
751 \\
636\end{array}$ & $\begin{array}{l}3839 \\
5241 \\
4175 \\
2695 \\
4745\end{array}$ & $\begin{array}{l}2394 \\
1414 \\
2081 \\
4083 \\
1462\end{array}$ & \\
\hline 36485 & 8847.882 & & -1851 & 244 & 4158 & 2868 & & & \\
\hline 37058 & 8847 & & 209 & 134 & -2835 & 685 & 4939 & 1845 & \\
\hline 50169 & & & 1297 & 74 & -846 & 1098 & 8059 & & \\
\hline & & & $\begin{array}{l}449 \\
916 \\
650\end{array}$ & & $\begin{array}{l}-330 \\
-233 \\
5955\end{array}$ & $\begin{array}{l}1775 \\
1544 \\
1794\end{array}$ & $\begin{array}{r}8274 \\
8596 \\
12041\end{array}$ & $\begin{array}{r}531 \\
719 \\
1724\end{array}$ & \\
\hline 70331 & & & & & -2142 & & & & \\
\hline 81009 & 9026.663 & 0.7 & 1909 & 209 & 11500 & 3045 & 15006 & 1487 & \\
\hline 83368 & & 883 & -195 & 280 & -59 & 4853 & & & \\
\hline 935 & & & 98 & & $\begin{array}{r}-975 \\
938\end{array}$ & & & & \\
\hline 94660 & & & $\begin{array}{l}-2048 \\
-1786\end{array}$ & & $\begin{array}{l}649 \\
252\end{array}$ & & $\begin{array}{l}8025 \\
9243\end{array}$ & & \\
\hline 3457 & 9026.793 & & 623 & 319 & 790 & 2646 & & & \\
\hline 6114 & 9026.761 & & -1923 & 113 & 1119 & 1192 & 219 & 165 & \\
\hline & & & & $\begin{array}{r}140 \\
72 \\
59 \\
104 \\
65\end{array}$ & $\begin{array}{r}174 \\
-514 \\
-36 \\
984 \\
-371\end{array}$ & $\begin{array}{r}1258 \\
616 \\
582 \\
934 \\
967\end{array}$ & & & \\
\hline & & & & & $\begin{array}{r}-18798 \\
6493\end{array}$ & & & & \\
\hline & & & $\begin{array}{r}922 \\
-2214\end{array}$ & & $\begin{array}{r}8070 \\
858\end{array}$ & & & & \\
\hline 120013 & & & $\begin{array}{r}628 \\
-2208 \\
1686\end{array}$ & & $\begin{array}{r}-1378 \\
4107 \\
5414\end{array}$ & $\begin{array}{l}2649 \\
4206 \\
2669\end{array}$ & & & \\
\hline & & & $\begin{array}{l}-348 \\
-410\end{array}$ & & $\begin{array}{r}2755 \\
-401\end{array}$ & $\begin{array}{r}2116 \\
706\end{array}$ & 3905 & 1855 & \\
\hline 134214 & & & $\begin{array}{l}-591 \\
-265\end{array}$ & & & & 4215 & 3219 & \\
\hline & $\begin{array}{l}8782.584 \\
8782.778 \\
8846.631 \\
8847.592 \\
9194.569\end{array}$ & & $\begin{array}{r}213 \\
247 \\
-377 \\
1533 \\
-214\end{array}$ & $\begin{array}{l}573 \\
398 \\
266 \\
292 \\
187\end{array}$ & $\begin{array}{r}11989 \\
9518 \\
-10956 \\
-25844 \\
5838\end{array}$ & $\begin{array}{l}6335 \\
7641 \\
7971 \\
6062 \\
6690\end{array}$ & $\begin{array}{l}25096 \\
28032 \\
23350 \\
26274 \\
23056\end{array}$ & $\begin{array}{l}2763 \\
2328 \\
7955 \\
8643 \\
5960\end{array}$ & \\
\hline
\end{tabular}

$\left(\left\langle H^{2}\right\rangle\right)^{1 / 2}$ still exceeds $\langle H\rangle$ by an amount characterizing the scatter of the local values of the field modulus around $\langle H\rangle$ across the stellar surface (see Paper V and Mathys 1993 for details).

The quadratic field is diagnosed from the second-order moment about the line centre of the unpolarized line profile, $R_{I}^{(2)}\left(\lambda_{0}\right)$. This moment characterizes the unpolarized

\begin{tabular}{|c|c|c|c|c|c|c|c|c|c|}
\hline HD & $\begin{array}{l}\text { HJD } \\
(2440000+)\end{array}$ & Phase & $\begin{array}{l}\text { Long. } \\
\text { field } \\
\qquad(\mathrm{G})\end{array}$ & $\sigma_{\mathrm{z}}$ & $\begin{array}{l}\text { Cross- } \\
\text { over } \\
\left(\mathrm{km} \mathrm{s}^{-}\right.\end{array}$ & $\begin{array}{r}\sigma_{\mathrm{x}} \\
-1 \\
\mathrm{G})\end{array}$ & $\begin{array}{l}\text { Quad, } \\
\text { field } \\
\text { (G }\end{array}$ & $\sigma_{\mathrm{q}}$ & \\
\hline 37909 & $\begin{array}{l}8845.531 \\
8846.465 \\
9194.475 \\
9195.464\end{array}$ & $\begin{array}{l}0.961 \\
0.012 \\
0.837 \\
0.890\end{array}$ & $\begin{array}{l}561 \\
588 \\
253 \\
423\end{array}$ & $\begin{array}{l}79 \\
45 \\
62 \\
53\end{array}$ & $\begin{array}{r}-568 \\
-1488 \\
-1012 \\
-1505\end{array}$ & & $\begin{array}{l}6158 \\
5801 \\
5028 \\
5326\end{array}$ & $\begin{array}{r}568 \\
736 \\
1197 \\
1149\end{array}$ & \\
\hline 137949 & & & $\begin{array}{l}1739 \\
1929\end{array}$ & & $\begin{array}{l}4818 \\
1730\end{array}$ & $\begin{array}{l}1467 \\
1177\end{array}$ & $\begin{array}{l}4724 \\
5047\end{array}$ & $\begin{array}{l}3265 \\
2548\end{array}$ & \\
\hline 144897 & 9026.879 & 0.794 & 2046 & 158 & 516 & 1754 & 9951 & 767 & \\
\hline 147010 & & & & & $\begin{array}{r}786 \\
12765\end{array}$ & & $\begin{array}{l}11701 \\
17348\end{array}$ & & \\
\hline 153882 & & $\begin{array}{l}0.158 \\
0.329 \\
0.272\end{array}$ & $\begin{array}{l}1335 \\
1289 \\
1383\end{array}$ & $\begin{array}{r}99 \\
424 \\
104\end{array}$ & $\begin{array}{l}2076 \\
7730 \\
1741\end{array}$ & $\begin{array}{l}2533 \\
1669 \\
1048\end{array}$ & $\begin{array}{l}5535 \\
3071 \\
3944\end{array}$ & $\begin{array}{l}1243 \\
2684 \\
1790\end{array}$ & \\
\hline 18107 & 8846.571 & & 1985 & 230 & 4153 & 4579 & 21028 & 827 & \\
\hline 65474 & & & $\begin{array}{r}169 \\
-55\end{array}$ & & $\begin{array}{r}-246 \\
-66\end{array}$ & & $\begin{array}{l}7577 \\
6457\end{array}$ & $\begin{array}{l}834 \\
685\end{array}$ & \\
\hline 166473 & & & $\begin{array}{l}-2036 \\
-2291 \\
-2110\end{array}$ & & $\begin{array}{r}-1241 \\
-631 \\
-2230\end{array}$ & $\begin{array}{r}1153 \\
1598 \\
845\end{array}$ & $\begin{array}{r}9935 \\
10774 \\
11210\end{array}$ & $\begin{array}{r}2091 \\
772 \\
573\end{array}$ & \\
\hline 168733 & $\begin{array}{l}8846 \\
8847 \\
9193 \\
9194\end{array}$ & & $\begin{array}{l}-594 \\
-819 \\
-632 \\
-439\end{array}$ & $\begin{array}{r}65 \\
125 \\
84 \\
117\end{array}$ & $\begin{array}{r}-183 \\
-350 \\
94 \\
-23\end{array}$ & $\begin{array}{r}1048 \\
1630 \\
656 \\
752\end{array}$ & $\begin{array}{l}2305 \\
3772 \\
1175\end{array}$ & & \\
\hline 175362 & $\begin{array}{l}8399.9 \\
8400.9 \\
8846.6 \\
8847.6 \\
9193.7 \\
9194.8\end{array}$ & $\begin{array}{l}0.257 \\
0.530 \\
0.868 \\
0.141 \\
0.352 \\
0.638\end{array}$ & $\begin{array}{r}-1699 \\
3565 \\
-3463 \\
-2045 \\
620 \\
2868\end{array}$ & $\begin{array}{l}367 \\
345 \\
404 \\
439 \\
195 \\
312\end{array}$ & $\begin{array}{r}-11887 \\
5074 \\
6158 \\
-7086 \\
-30559 \\
3470\end{array}$ & $\begin{array}{r}5347 \\
8048 \\
5000 \\
3126 \\
5770 \\
12796\end{array}$ & $\begin{array}{l}15339 \\
20019 \\
18449 \\
11046 \\
16616\end{array}$ & $\begin{array}{l}3779 \\
2969 \\
3016 \\
6076\end{array}$ & \\
\hline 176232 & 8782.764 & & 187 & 178 & -812 & 1417 & 3014 & 2781 & \\
\hline 187474 & $\begin{array}{l}83 \\
87 \\
88 \\
91\end{array}$ & & $\begin{array}{r}-771 \\
1644 \\
1265 \\
1461\end{array}$ & $\begin{array}{r}122 \\
107 \\
79 \\
124\end{array}$ & $\begin{array}{r}860 \\
2923 \\
777 \\
-80\end{array}$ & $\begin{array}{r}1216 \\
1422 \\
882 \\
1400\end{array}$ & $\begin{array}{l}7627 \\
7760 \\
5841 \\
5584\end{array}$ & $\begin{array}{r}1583 \\
1312 \\
463 \\
1107\end{array}$ & \\
\hline & & & & & $\begin{array}{r}3849 \\
1465 \\
768\end{array}$ & & & & \\
\hline 3756 & 8782.804 & & -467 & 241 & 3046 & 3457 & 5221 & 8444 & \\
\hline 35238 & 919 & & -1338 & 247 & -5336 & 1568 & 10551 & 1236 & \\
\hline 201601 & & & $\begin{array}{l}-1037 \\
-1133 \\
-1083 \\
-1081\end{array}$ & $\begin{array}{r}99 \\
143 \\
53 \\
68\end{array}$ & $\begin{array}{r}-688 \\
-1618 \\
471 \\
-95\end{array}$ & $\begin{array}{r}1109 \\
1170 \\
474 \\
753\end{array}$ & $\begin{array}{l}5206 \\
4956\end{array}$ & $\begin{array}{l}1453 \\
1005\end{array}$ & $\begin{array}{l}23 \\
18\end{array}$ \\
\hline 203932 & & & $\begin{array}{r}-322 \\
146\end{array}$ & & $\begin{array}{r}1567 \\
428\end{array}$ & $\begin{array}{r}1459 \\
598\end{array}$ & & & \\
\hline 216018 & $\begin{array}{l}8846.744 \\
8847.748 \\
9193.897\end{array}$ & & $\begin{array}{l}1323 \\
1358 \\
1265\end{array}$ & $\begin{array}{r}103 \\
99 \\
93\end{array}$ & $\begin{array}{r}-571 \\
-160 \\
631\end{array}$ & $\begin{array}{r}1025 \\
1080 \\
777\end{array}$ & $\begin{array}{l}7140 \\
6787 \\
7251\end{array}$ & $\begin{array}{r}647 \\
1310 \\
1768\end{array}$ & \\
\hline 217522 & 8845.809 & & -394 & 124 & 376 & 788 & 4955 & 2030 & \\
\hline 218495 & 8847.842 & & -606 & 280 & 3817 & 1642 & & & \\
\hline
\end{tabular}

line width. The quadratic field determination rests on the relation:

$R_{I}^{(2)}\left(\lambda_{0}\right)=C+3 / 4\left(S_{2}+D_{2} / 3\right) \Delta \lambda_{\mathrm{Z}}^{2}\left(\left\langle H^{2}\right\rangle+\left\langle H_{\mathrm{z}}^{2}\right\rangle\right)^{1 / 2}$

$S_{2}$ and $D_{2}$ are atomic parameters obtained from linear combinations of the effective Landé factor and of the second-order moments of the $\pi$ and $\sigma$ components of the transition about their respective centre. They characterize the total spread of the Zeeman pattern and its anomalous 
character. Their expression in terms of the Landé factors and of the total angular momentum quantum numbers of the atomic levels between which the transition takes place has been established by Mathys (1988) and Mathys \& Stenflo $(1987 \mathrm{a}, \mathrm{b}) . C$ is a constant accounting for the non-magnetic part of the unpolarized line width (including contributions such as natural width, rotational and thermal Doppler broadening, instrumental profile, etc.).

Again, the actual determination of the quadratic field is carried out through measuring $R_{I}^{(2)}\left(\lambda_{0}\right)$ for a sample of lines and performing a weighted linear regression of the form given in Eq. (3) over this set of measurements. Contrary to the cases of the longitudinal field and of the crossover, this regression is not forced through the origin. Instead, two free parameters are determined from it: the quadratic field and the constant $C$. In Paper V, for stars repeatedly observed at various rotation phases, it was assumed that $C$ is phase-independent: accordingly, a single value of this constant was derived from the simultaneous consideration of all the observations of the considered star, which allowed a better accuracy to be achieved. Here, this approach can no longer be used, because of the inhomogeneity of the data (resulting from the use of different instrumental configurations). As a result, the accuracy of the present determinations of both $C$ and $\left(\left\langle H^{2}\right\rangle+\left\langle H_{\mathrm{z}}^{2}\right\rangle\right)^{1 / 2}$ is less good than in Paper V, even when these determinations rely on spectra of better resolution.

\section{Results}

\subsection{Generalities}

Magnetic field moments have been derived as described in the previous section from 95 observations of 44 stars. These measurements are presented in Table 3 . The columns give, in order: the star HD (or HDE) number, the heliocentric Julian date of mid-observation, the rotation phase (for stars whose rotation period is reliably known), the longitudinal field $\left\langle H_{\mathrm{z}}\right\rangle$ and its estimated uncertainty $\sigma_{\mathrm{z}}$, the crossover $v \sin i\left\langle x H_{\mathrm{z}}\right\rangle$ and its estimated uncertainty $\sigma_{\mathrm{x}}$, the quadratic field $\left(\left\langle H^{2}\right\rangle+\left\langle H_{\mathrm{z}}^{2}\right\rangle\right)^{1 / 2}$ and its estimated uncertainty $\sigma_{\mathrm{q}}$, and the number of spectral lines used for the determination of the field moments. The absence of entries in the last two columns corresponds to cases when the fit of the measurements of $R_{I}^{(2)}\left(\lambda_{0}\right)$ by a function of the form given in Eq. (3) yields a negative value of $\left(\left\langle H^{2}\right\rangle+\left\langle H_{\mathrm{z}}^{2}\right\rangle\right)$. As discussed in Paper $\mathrm{V}$, this may happen when the square field is small compared to the accuracy which can be achieved in its diagnosis.

For the stars for which observations have been obtained repeatedly throughout the rotation cycle (including observations already reported in Papers III to V), the variations of the field moments can be well represented either by a cosine wave:

$A_{\mathrm{H}}(\phi)=A_{0}+A_{1} \cos \left[2 \pi\left(\phi-\phi_{1}\right)\right]$, or by the superposition of a cosine and of its first harmonic:

$$
\begin{aligned}
A_{\mathrm{H}}(\phi)= & A_{0}+A_{1} \cos \left[2 \pi\left(\phi-\phi_{1}\right)\right] \\
& +A_{2} \cos \left[2 \pi\left(2 \phi-\phi_{2}\right)\right] .
\end{aligned}
$$

In these expressions, $A_{\mathrm{H}}$ stands for any of the measured quantities $\left\langle H_{z}\right\rangle, v \sin i\left\langle x H_{z}\right\rangle$, or $\left(\left\langle H^{2}\right\rangle+\left\langle H_{z}^{2}\right\rangle\right)^{1 / 2}$. $\phi$ is the rotation phase, determined using the values of the period $P$ and of the Julian date $\mathrm{HJD}_{0}$ of the phase origin which appear in Table 2 . The mean value $A_{0}$, the amplitude(s) $A_{1}$ (and when significant $A_{2}$ ), and the phase(s) $\phi_{1}$ (and possibly $\phi_{2}$ ) of the variations are derived through a leastsquares fit of the field moment measurements by function (4) or (5). This fit is weighted according to the uncertainty of the individual measurements.

The results of those fits are presented in Table 4 (for $\left\langle H_{\mathrm{z}}\right\rangle$; the amplitudes are denoted $H_{i}, i=0,2$, and the phases $\phi_{H_{i}}, i=1,2$ ), Table 5 (for $v \sin i\left\langle x H_{z}\right\rangle$, amplitudes $X_{i}$ and phases $\phi_{X_{i}}$ ), and Table 6 (for $\left(\left\langle H^{2}\right\rangle+\right.$ $\left.\left\langle H_{\mathrm{z}}^{2}\right\rangle\right)^{1 / 2}$, amplitudes $Q_{i}$ and phases $\left.\phi_{Q_{i}}\right)$. Columns 7 to 9 of these tables give the number of degrees of freedom about the fit $\nu$, the reduced $\chi^{2}$ of the fit $\chi^{2} / \nu$, and the multiple correlation coefficient $R$. Graphical representations of the fits also appear in several of the phase diagrams plotted below. In those diagrams, the meaning of the symbols used to represent the data points is as follows. Filled squares correspond to our measurements of Papers III to V. Our new data appear either as open squares (for those obtained with the long camera of CASPEC) or as filled triangles (corresponding to observations performed with the short camera of CASPEC). When data of other authors are included in the figures for comparison, crosses are used to distinguish them. As in the previous papers of this series, the error bars shown for our measurements correspond to $\pm 1 \sigma$.

The results obtained will now be discussed star by star.

\subsection{Stars already studied in Papers II to V}

\subsubsection{HD 24712}

Only 2 observations of the rapidly oscillating Ap (roAp) star HD 24712 were discussed in the previous papers of this series. Five new observations have now been obtained. The resulting 7 measurements of the longitudinal field are plotted together with Preston's (1972) data in Fig. 1, against the phases computed from the value of the rotation period (12. 4572) favoured by Kurtz \& Marang (1987). The phase diagram shown in Fig. 2 rests on the value of the period (12.4610) proposed in Paper II. As suspected in that paper, comparison of Figs. 1 and 2 strongly supports the view that the actual value of the rotation period is (close to) 12 d 4610. It has been shown in Paper II that this value is also consistent with 7 unpublished $\left\langle H_{\mathrm{z}}\right\rangle$ measurements 
Table 4. Variation of the longitudinal field: least-squares fit parameters

\begin{tabular}{|c|c|c|c|c|c|c|c|c|}
\hline Star & $\begin{array}{l}H_{0} \pm \sigma \\
\quad(\mathrm{G})\end{array}$ & $\begin{array}{l}H_{1} \pm \sigma \\
\quad(\mathrm{G})\end{array}$ & $\phi_{H_{1}} \pm \sigma$ & $\begin{array}{l}H_{2} \pm \sigma \\
(\mathrm{G})\end{array}$ & $\phi_{H_{2}} \pm \sigma$ & $\nu$ & $\chi^{2} / \nu$ & $R$ \\
\hline HD 83368 & $-17 \pm 74$ & $576 \pm 100$ & $0.496 \pm 0.027$ & - & - & 10 & 1.7 & 0.88 \\
\hline HD 116458 & $-1724 \pm 65$ & $420 \pm 103$ & $0.490 \pm 0.036$ & - & - & 7 & 3.5 & 0.89 \\
\hline HD 119419 & $\begin{array}{l}-759 \pm 93 \\
-696 \pm 76\end{array}$ & $\begin{array}{l}2022 \pm 124 \\
2181 \pm 109\end{array}$ & $\begin{array}{l}0.584 \pm 0.010 \\
0.585 \pm 0.008\end{array}$ & $382 \pm 107$ & $\begin{aligned} & - \\
0.386 & \pm 0.045\end{aligned}$ & $\begin{array}{l}19 \\
17\end{array}$ & $\begin{array}{l}1.3 \\
0.8\end{array}$ & $\begin{array}{l}0.96 \\
0.98\end{array}$ \\
\hline HD 125248 & $105 \pm 65$ & $1996 \pm 87$ & $0.055 \pm 0.007$ & - & - & 18 & 2.5 & 0.98 \\
\hline HD 126515 & $-301 \pm 249$ & $2306 \pm 227$ & $0.409 \pm 0.021$ & $515 \pm 336$ & $0.540 \pm 0.088$ & 4 & 1.5 & 0.99 \\
\hline HD 137509 & $\begin{array}{l}166 \pm 159 \\
302 \pm 65\end{array}$ & $\begin{array}{l}895 \pm 243 \\
803 \pm 94\end{array}$ & $\begin{array}{l}0.677 \pm 0.037 \\
0.685 \pm 0.016\end{array}$ & $\begin{array}{c}- \\
809 \pm 98\end{array}$ & $0.449 \stackrel{-}{ \pm} 0.015$ & $\begin{array}{r}11 \\
9\end{array}$ & $\begin{array}{l}3.0 \\
0.4\end{array}$ & $\begin{array}{l}0.78 \\
0.98\end{array}$ \\
\hline HD 137909 & $-223 \pm 27$ & $811 \pm 30$ & $0.982 \pm 0.007$ & - & - & 12 & 0.5 & 0.99 \\
\hline HD 147010 & $\begin{array}{l}-3250 \pm 133 \\
-3380 \pm 105\end{array}$ & $\begin{array}{l}1150 \pm 167 \\
1102 \pm 125\end{array}$ & $\begin{array}{l}0.805 \pm 0.026 \\
0.762 \pm 0.024\end{array}$ & $\begin{array}{c}- \\
459 \pm 152\end{array}$ & $\begin{aligned} & - \\
0.588 & \pm 0.052\end{aligned}$ & $\begin{array}{l}16 \\
14\end{array}$ & $\begin{array}{l}2.9 \\
1.6\end{array}$ & $\begin{array}{l}0.87 \\
0.94\end{array}$ \\
\hline HD 153882 & $136 \pm 54$ & $1382 \pm 71$ & $0.221 \pm 0.009$ & - & - & 14 & 1.5 & 0.98 \\
\hline HD 175362 & $\begin{array}{l}-678 \pm 152 \\
-432 \pm 113\end{array}$ & $\begin{array}{l}4499 \pm 235 \\
4259 \pm 164\end{array}$ & $\begin{array}{l}0.513 \pm 0.008 \\
0.507 \pm 0.006\end{array}$ & $955 \overline{-}=207$ & $0.092 \pm 0.027$ & $\begin{array}{l}27 \\
25\end{array}$ & $\begin{array}{l}4.8 \\
2.2\end{array}$ & $\begin{array}{l}0.97 \\
0.99\end{array}$ \\
\hline HD 187474 & $-216 \pm 183$ & $2156 \pm 216$ & $0.982 \pm 0.011$ & - & - & 8 & 5.2 & 0.96 \\
\hline
\end{tabular}

Table 5. Variation of the crossover: least-squares fit parameters

\begin{tabular}{|c|c|c|c|c|c|c|c|c|}
\hline Star & $\begin{array}{c}X_{0} \pm \sigma \\
\left(\mathrm{km} \mathrm{s}^{-1} \mathrm{G}\right)\end{array}$ & $\begin{array}{c}X_{1} \pm \sigma \\
\left(\mathrm{km} \mathrm{s}^{-1} \mathrm{G}\right)\end{array}$ & $\phi_{X_{1}} \pm \sigma$ & $\begin{array}{c}X_{2} \pm \sigma \\
\left(\mathrm{kms}^{-1} \mathrm{G}\right)\end{array}$ & $\phi_{X_{2}} \pm \sigma$ & $\nu$ & $\chi^{2} / \nu$ & $R$ \\
\hline HD 83368 & $2021 \pm 1506$ & $10895 \pm 2076$ & $0.775 \pm 0.031$ & - & - & 10 & 2.3 & 0.86 \\
\hline HD 125248 & $748 \pm 530$ & $6642 \pm 749$ & $0.298 \pm 0.017$ & - & - & 18 & 0.9 & 0.90 \\
\hline HD 137509 & $\begin{array}{r}-11804 \pm 4871 \\
-4240 \pm 2978\end{array}$ & $\begin{array}{l}15200 \pm 7223 \\
12429 \pm 3947\end{array}$ & $\begin{array}{l}0.072 \pm 0.081 \\
0.090 \pm 0.052\end{array}$ & $24263 \stackrel{-}{ \pm} 4051$ & $0.603 \pm 0.026$ & $\begin{array}{r}11 \\
9\end{array}$ & $\begin{array}{l}5.0 \\
1.3\end{array}$ & $\begin{array}{l}0.62 \\
0.93\end{array}$ \\
\hline HD 137909 & $-19 \pm 363$ & $1941 \pm 576$ & $0.286 \pm 0.039$ & - & - & 12 & 1.5 & 0.75 \\
\hline HD 147010 & $\begin{array}{l}1560 \pm 1272 \\
1753 \pm 1085\end{array}$ & $\begin{array}{l}4784 \pm 1786 \\
4603 \pm 1405\end{array}$ & $\begin{array}{l}0.855 \pm 0.062 \\
0.804 \pm 0.055\end{array}$ & $4403 \pm 1679$ & $0.705 \stackrel{-}{ \pm 0.062}$ & $\begin{array}{l}16 \\
14\end{array}$ & $\begin{array}{l}1.5 \\
0.9\end{array}$ & $\begin{array}{l}0.54 \\
0.78\end{array}$ \\
\hline HD 153882 & $1437 \pm 913$ & $12585 \pm 1435$ & $0.500 \pm 0.015$ & - & - & 14 & 1.8 & 0.92 \\
\hline HD 175362 & $\begin{array}{r}3590 \pm 3525 \\
576 \pm 2345\end{array}$ & $\begin{array}{l}29545 \pm 4924 \\
34374 \pm 3321\end{array}$ & $\begin{array}{l}0.734 \pm 0.028 \\
0.720 \pm 0.016\end{array}$ & $22291 \pm 4107$ & $0.382 \stackrel{-}{ \pm} 0.028$ & $\begin{array}{l}27 \\
25\end{array}$ & $\begin{array}{l}5.6 \\
2.4\end{array}$ & $\begin{array}{l}0.77 \\
0.92\end{array}$ \\
\hline
\end{tabular}

Table 6. Variation of the quadratic field: least-squares fit parameters

\begin{tabular}{|c|c|c|c|c|c|c|c|c|}
\hline Star & $\begin{array}{c}Q_{0} \pm \sigma \\
(\mathrm{G})\end{array}$ & $\begin{array}{l}Q_{1} \pm \sigma \\
\quad(\mathrm{G})\end{array}$ & $\phi_{Q_{1}} \pm \sigma$ & $\begin{array}{c}Q_{2} \pm \sigma \\
(\mathrm{G})\end{array}$ & $\phi_{Q_{2}} \pm \sigma$ & $\nu$ & $\chi^{2} / \nu$ & $R$ \\
\hline HD 83368 & $11375 \pm 359$ & $1453 \pm 480$ & $0.690 \pm 0.060$ & - & - & 9 & 0.7 & 0.72 \\
\hline HD 116458 & $6222 \pm 244$ & $1003 \pm 431$ & $0.979 \pm 0.059$ & - & - & 7 & 1.9 & 0.74 \\
\hline HD 119419 & $\begin{array}{l}23356 \pm 434 \\
23032 \pm 488\end{array}$ & $\begin{array}{l}2256 \pm 589 \\
2565 \pm 625\end{array}$ & $\begin{array}{l}0.984 \pm 0.047 \\
0.011 \pm 0.045\end{array}$ & $1053 \pm 706$ & $\begin{array}{c}- \\
0.779 \pm 0.092\end{array}$ & $\begin{array}{l}19 \\
17\end{array}$ & $\begin{array}{l}0.6 \\
0.6\end{array}$ & $\begin{array}{l}0.68 \\
0.72\end{array}$ \\
\hline HD 125248 & $9844 \pm 161$ & $1429 \pm 215$ & $0.647 \pm 0.024$ & - & - & 18 & 1.2 & 0.84 \\
\hline HD 126515 & $17126 \pm 295$ & $2788 \pm 420$ & $0.951 \pm 0.031$ & - & - & 6 & 0.5 & 0.97 \\
\hline HD 137509 & $\begin{array}{l}31160 \pm 678 \\
29892 \pm 955\end{array}$ & $\begin{array}{l}3783 \pm 969 \\
3514 \pm 1182\end{array}$ & $\begin{array}{l}0.515 \pm 0.040 \\
0.607 \pm 0.054\end{array}$ & $\begin{array}{c}- \\
1978 \pm 1157\end{array}$ & $\begin{array}{c}- \\
0.179 \pm 0.080\end{array}$ & $\begin{array}{r}11 \\
9\end{array}$ & $\begin{array}{l}1.1 \\
0.8\end{array}$ & $\begin{array}{l}0.82 \\
0.90\end{array}$ \\
\hline HD 137909 & $6079 \pm 182$ & $1197 \pm 325$ & $0.230 \pm 0.031$ & - & - & 12 & 0.5 & 0.73 \\
\hline HD 147010 & $\begin{array}{l}14348 \pm 181 \\
14381 \pm 157\end{array}$ & $\begin{array}{l}2227 \pm 257 \\
2120 \pm 213\end{array}$ & $\begin{array}{l}0.869 \pm 0.019 \\
0.861 \pm 0.016\end{array}$ & $601 \pm 261$ & $\begin{array}{c}- \\
0.676 \pm 0.065\end{array}$ & $\begin{array}{l}16 \\
14\end{array}$ & $\begin{array}{l}0.8 \\
0.5\end{array}$ & $\begin{array}{l}0.90 \\
0.94\end{array}$ \\
\hline HD 153882 & $5866 \pm 315$ & - & - & $1546 \pm 415$ & $0.024 \pm 0.046$ & 14 & 1.3 & 0.71 \\
\hline HD 175362 & $\begin{array}{l}16219 \pm 301 \\
15058 \pm 292\end{array}$ & $\begin{array}{l}3889 \pm 402 \\
4209 \pm 421\end{array}$ & $\begin{array}{l}0.506 \pm 0.018 \\
0.510 \pm 0.016\end{array}$ & $\begin{array}{c}- \\
770 \pm 390\end{array}$ & $0.686 \pm 0.124$ & $\begin{array}{l}26 \\
24\end{array}$ & $\begin{array}{l}0.5 \\
0.5\end{array}$ & $\begin{array}{l}0.89 \\
0.91\end{array}$ \\
\hline
\end{tabular}




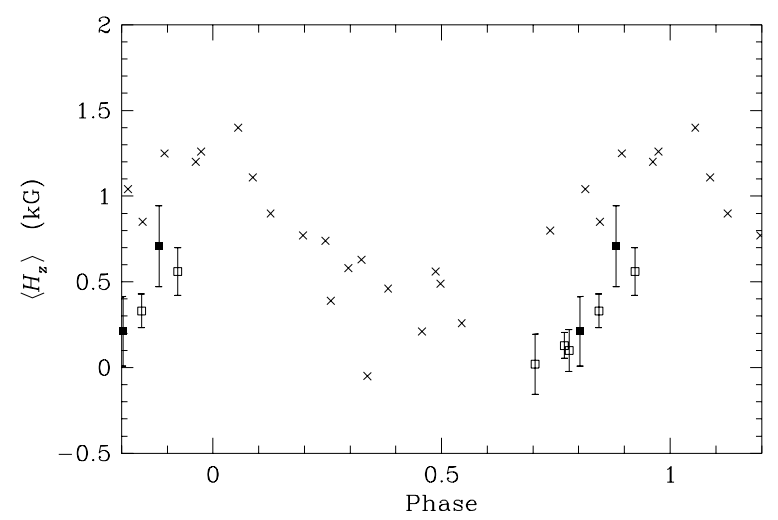

Fig. 1. Plot of our measurements of the longitudinal field of HD 24712 and of those of Preston (1972) against the rotation phase computed assuming that the rotation period is $12 \mathrm{~d} .4572$ (see the text for the meaning of the symbols)

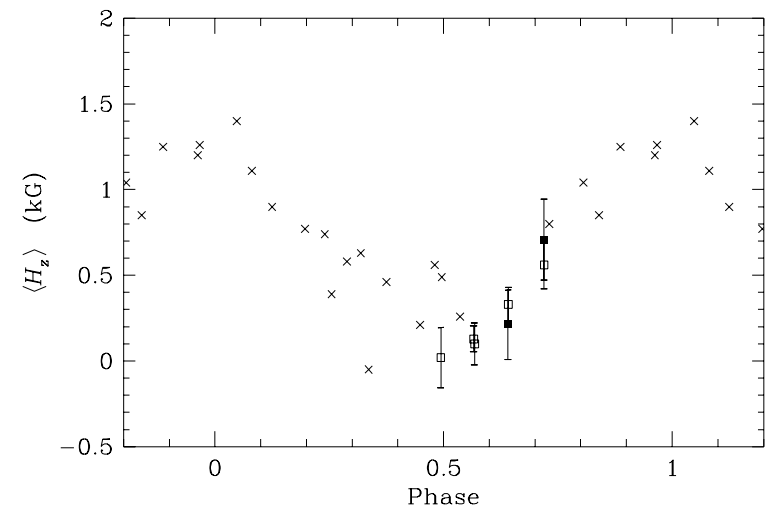

Fig. 2. Same as Fig. 1, but assuming a value of 12.4610 for the rotation period

of J.D. Landstreet (private communication). Reasons for the possible inaccuracy of the value derived by Kurtz \& Marang (1987) have been discussed in Paper II.

Crossover and quadratic field remain below the detection limit in HD 24712, not surprisingly given its low $v \sin i$ and the small upper limit of its field modulus (Mathys \& Lanz 1992).

\subsubsection{HD 83368}

HD 83368 is also a roAp star. Its fairly rapid rotation $\left(v \sin i=33 \mathrm{~km} \mathrm{~s}^{-1}\right)$ makes magnetic field diagnosis difficult. This accounts for our present inability to derive a quadratic field from our only new observation of this star, and for the rather poor accuracy of the crossover determination reported here. The latter, however, is not inconsistent with our previous data, as can be seen in Fig. 3. The same figure also shows that our new $\left\langle H_{\mathrm{z}}\right\rangle$ measurement agrees quite well with our 12 earlier determinations. We confirm that, to the achieved accuracy, the curves of variation of the three field moments discussed in this paper

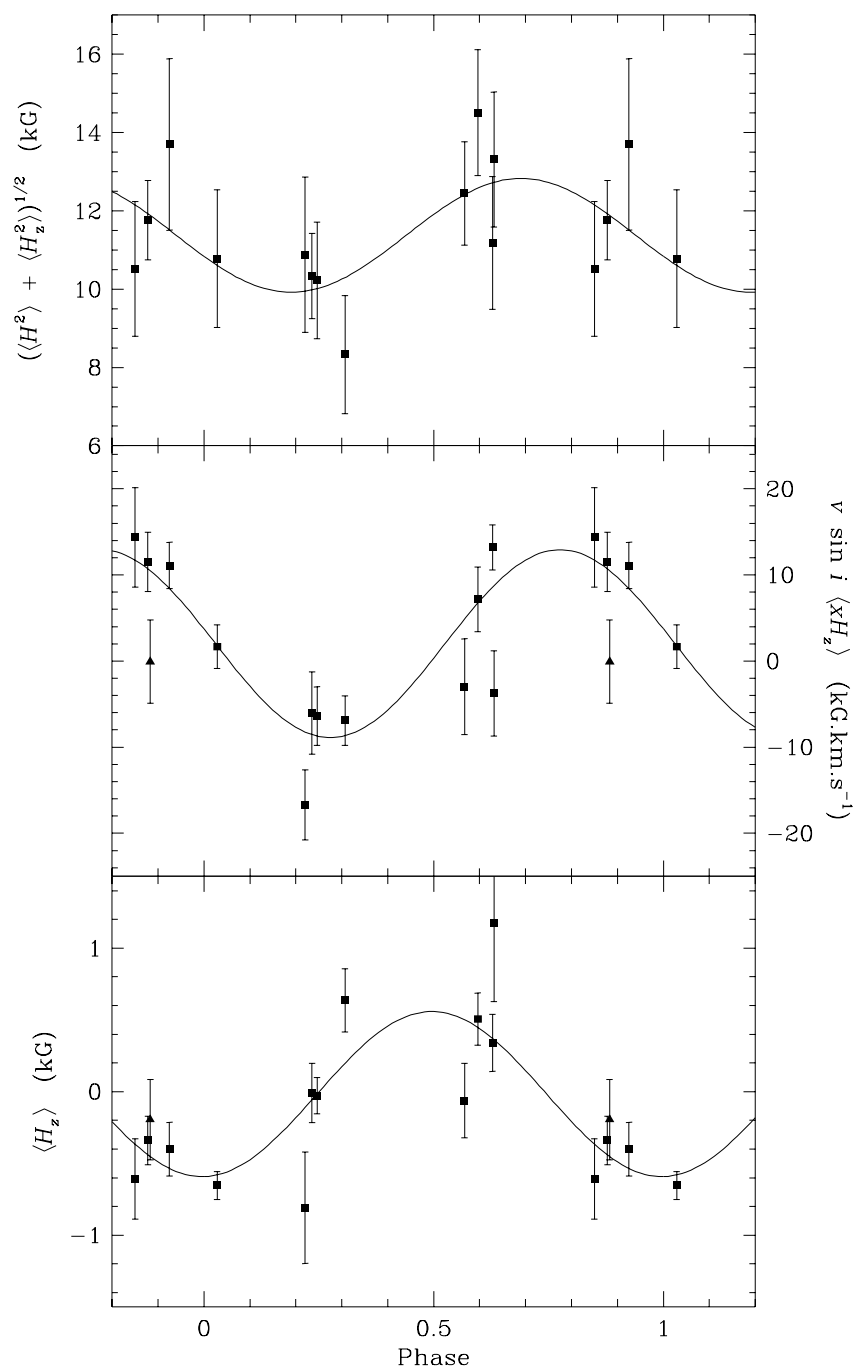

Fig. 3. Phase diagram of our measurements of the mean longitudinal magnetic field (lower panel), of the crossover (centre), and of the mean quadratic magnetic field (upper panel) of HD 83368 (see the text for the meaning of the symbols). The curves are least-squares fits of the data by sinusoids

do not significantly depart from sinusoids. Nevertheless, the variations of the quadratic field are at the limit of significance, and their shape cannot be regarded as well established.

\subsubsection{HD 94660}

Resolved magnetically split lines have been discovered in HD 94660 by Mathys (1990). On the basis of photometric observations, Hensberge (1993) suggested that the rotation period of this star may be close to $2700 \mathrm{~d}$. This is well supported by the mean magnetic field modulus measurements of Mathys et al. (1996; hereafter MHLLM). The latter authors also argued that no longitudinal field variations are detected either in our own measurements (2 in 
Paper III and 2 here) or in those of other authors (Borra \& Landstreet 1975; Bohlender et al. 1993). Given the good accuracy (of the order of $100 \mathrm{G}$ ) of our $4\left\langle H_{\mathrm{z}}\right\rangle$ determinations and their reasonable distribution in phase (assuming that the rotation period is indeed close to $2700 \mathrm{~d}$ ), it appears unlikely that $\left\langle H_{\mathrm{z}}\right\rangle$ may vary with a peak-to-peak amplitude exceeding 300 or $400 \mathrm{G}$. This is amazing, considering that the peak-to-peak amplitude of variation of the mean field modulus $\langle H\rangle$ of HD 94660 must be about $300 \mathrm{G}$ or larger, and that large variations of $\langle H\rangle$ are seldom observed, even in stars where $\left\langle H_{z}\right\rangle$ is strongly variable and/or undergoes polarity reversal. In fact, HD 94660 appears as a unique case of a star where one observes definite variations of $\langle H\rangle$ but not of $\left\langle H_{\mathrm{z}}\right\rangle$. This, consistently with the very anharmonic shape of the field modulus variations (see Fig. 26 of MHLLM) hints at a rather unusual geometrical structure of the magnetic field.

We neither detect any significant variation of the quadratic field. This is not unexpected: the relative amplitude of the latter should be at most of the same order as the relative amplitude of variation of the field modulus, that is, about $5 \%$. Such variations are below the detection limit at the accuracy achieved in the quadratic field diagnosis. Note also that the ratio between $\left(\left\langle H^{2}\right\rangle+\left\langle H_{\mathrm{z}}^{2}\right\rangle\right)^{1 / 2}$ and $\langle H\rangle$ is approximately 1.3 .

Finally, crossover can, of course, not be measured in a star rotating so slowly.

\subsubsection{HD 98457}

Our attention had been called to HD 98457 by its unusually large photometric variations (Waelkens 1985). Our first two observations of this star, obtained at phases 0.152 and 0.983 , had not allowed us to detect definitely a magnetic field from the consideration of any of the three field moments that we are studying (Papers II to V), although values of the quadratic field were measured at the 2.6 and $2.9 \sigma$ levels. We have obtained one new observation, at phase 0.446 (the uncertainty of the relative phasing with respect to our previous measurements should be less than 0.03). Again, we do not detect any significant longitudinal field or crossover, while we are unable to diagnose the quadratic field. Given the improvement of the phase sampling resulting from this new observation, it seems increasingly unlikely that HD 98457 may have a very strong magnetic field.

\subsubsection{HD 116458}

Like HD 94660, HD 116458 is a star in which magnetically resolved lines have been discovered by Mathys (1990). The rotation period, $P=147 . \mathrm{d} 9$, has been determined from photometry by Hensberge (1993), who has shown that it is consistent with our 5 measurements of $\left\langle H_{z}\right\rangle$ presented in Paper II. These are complemented here by 5 new determinations. All our longitudinal field measurements are

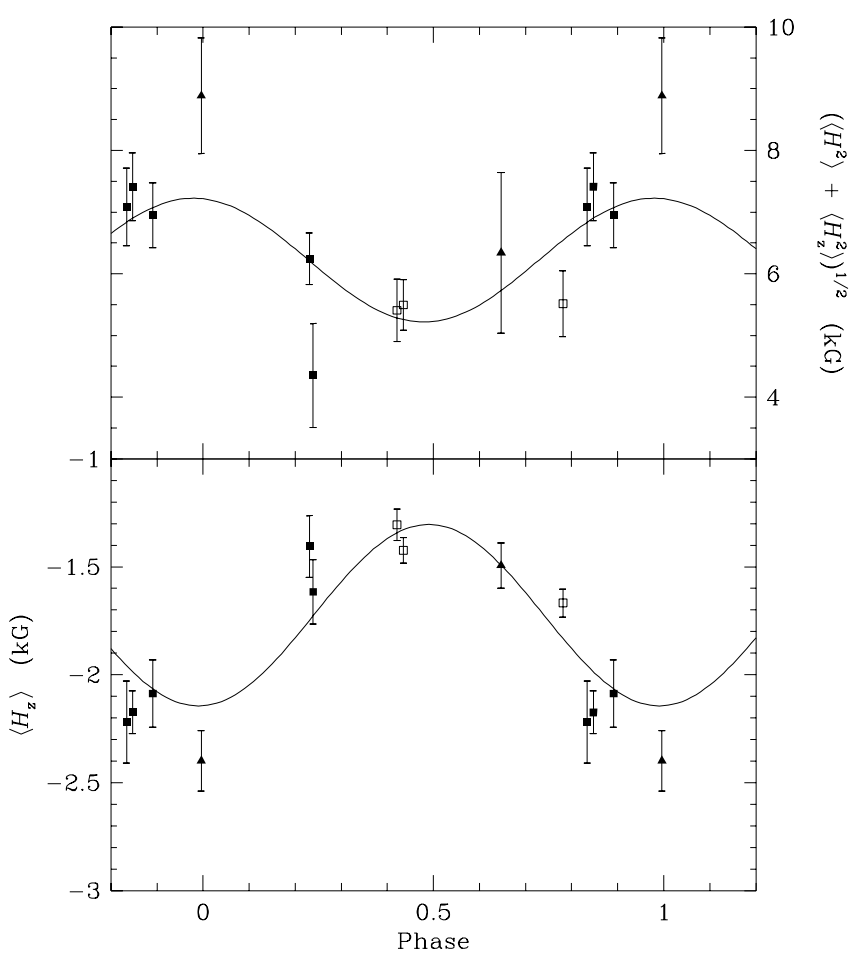

Fig. 4. Phase diagram of our measurements of the mean longitudinal magnetic field (lower panel) and of the mean quadratic magnetic field (upper panel) of HD 116458 (see the text for the meaning of the symbols). The curves are least-squares fits of the data by sinusoids

plotted against rotation phase in Fig. 4. The variations have a fairly small amplitude but are undisputable. The reduced $\chi^{2}$ obtained when fitting them by a cosine curve is somewhat high, but there is no definite indication of anharmonicity.

HD 116458 rotates too slowly to allow detection of any crossover.

From the consideration of the upper panel of Fig. 4, the quadratic field of HD 116458 shows some hint of variability. However the amplitude of the corresponding sinusoidal fit is at most marginally significant (see Table 6). As a matter of fact, the field modulus of this star is not detectably variable (MHLLM), so that we do not expect $\left\langle H^{2}\right\rangle$ to be variable either. Any variation of $\left(\left\langle H^{2}\right\rangle+\left\langle H_{\mathrm{z}}^{2}\right\rangle\right)^{1 / 2}$ should thus arise from the contribution of $\left\langle H_{\mathrm{z}}^{2}\right\rangle$. It can also be noted that the ratio between the mean value $Q_{0}$ of the quadratic field and the mean magnetic field modulus (see Table 3 of MHLLM) is 1.33.

\subsubsection{HD 119419}

Two new observations of HD 119419 have been obtained. When plotted together with our 20 previous measurements against the phase computed with the period 2. 6006 (Lanz \& Mathys 1991), the two new data show a definite phase shift, indicating that the period is no longer 


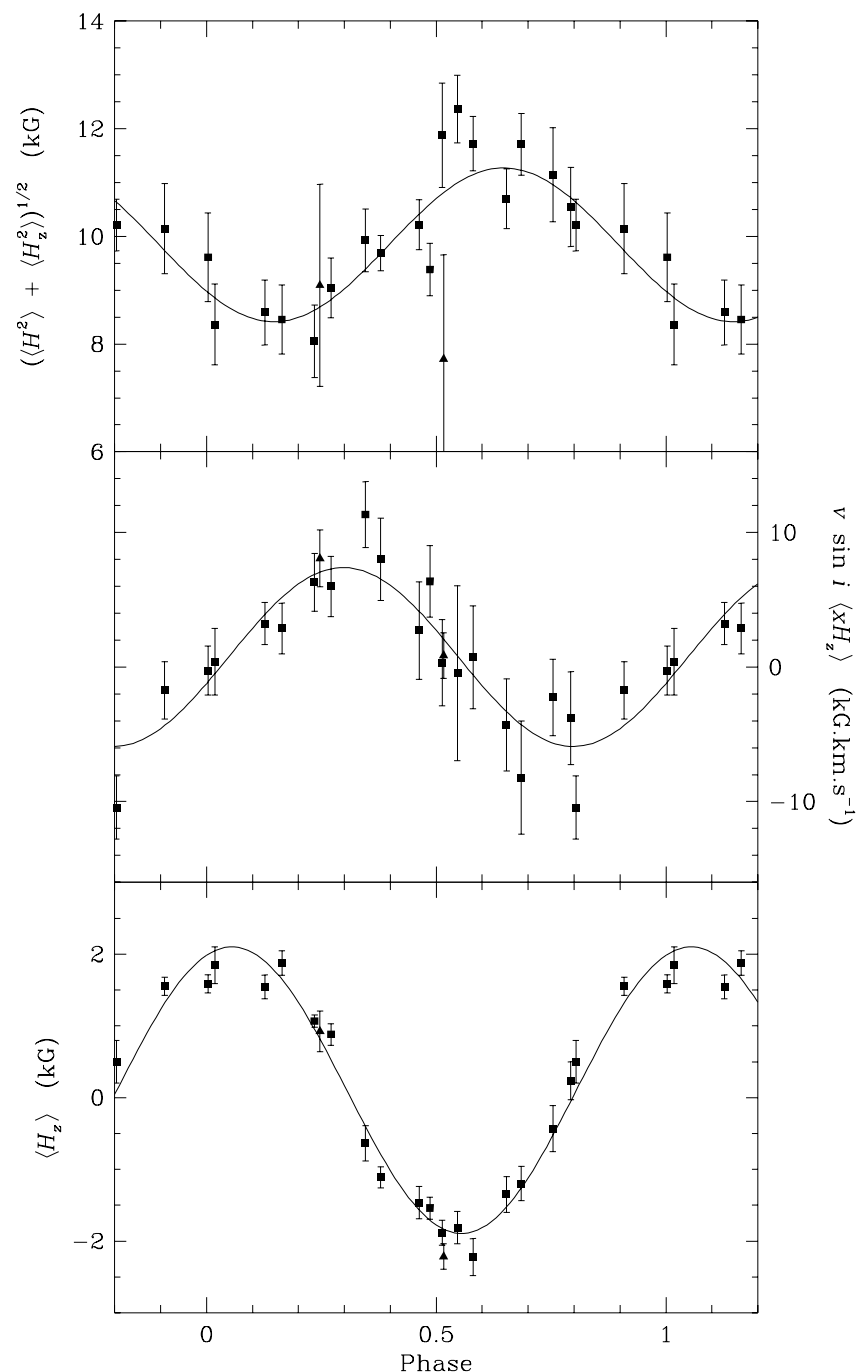

Fig. 6. Phase diagram of our measurements of the mean longitudinal magnetic field (lower panel), of the crossover (centre), and of the mean quadratic magnetic field (upper panel) of HD 125248 (see the text for the meaning of the symbols). The curves are least-squares fits of the data by sinusoids

the ratio $\left(\left\langle H^{2}\right\rangle+\left\langle H_{\mathrm{z}}^{2}\right\rangle\right)^{1 / 2} /\langle H\rangle$ significantly varies along the rotation cycle, ranging approximately from 1.25 at field modulus maximum to close to 1.50 at field modulus minimum. This indicates that, at least at some phases, the contribution of $\left\langle H_{\mathrm{z}}^{2}\right\rangle$ to the quadratic field must be rather considerable.

Crossover is not detected in HD 126515 as a result of its slow rotation.

\subsubsection{HD 128898}

After many unsuccessful attempts by various authors, the rotation period of HD 128898, the brightest of all roAp stars known, has been determined by Kurtz et al. (1994): $P=4$ d 4790 . Using this value of the period, our 2 new

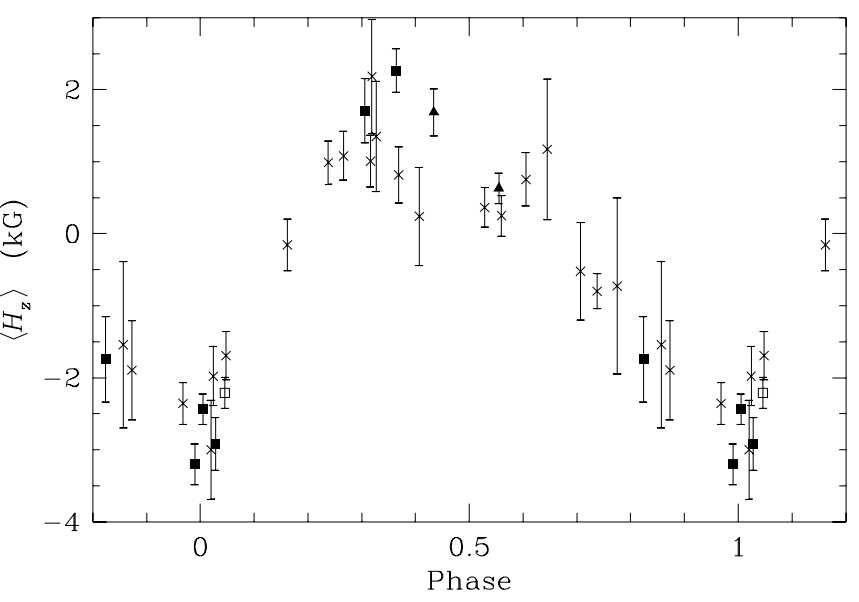

Fig. 7. Phase diagram of our measurements of the longitudinal field of HD 126515 and of unpublished determinations of Bohlender \& Hill (see the text for the meaning of the symbols)

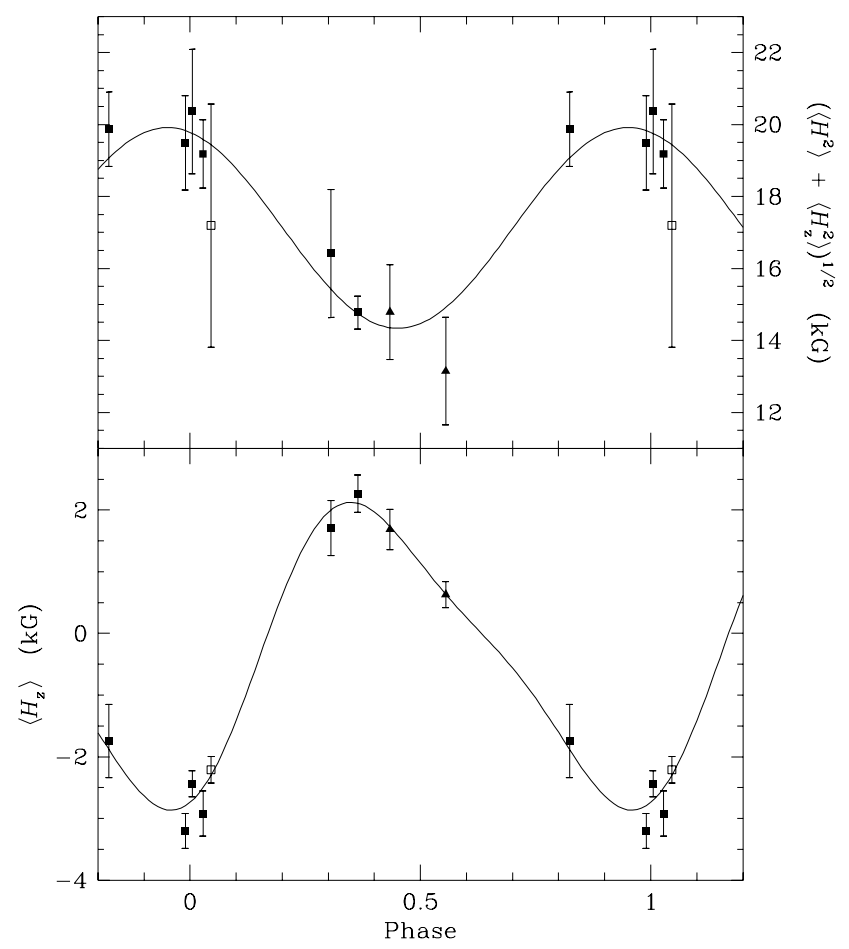

Fig. 8. Phase diagram of our measurements of the mean longitudinal magnetic field (lower panel) and of the mean quadratic magnetic field (upper panel) of HD 126515 (see the text for the meaning of the symbols). The curves are least-squares fits of the data by a cosine wave and its first harmonic (for the longitudinal field) and by a cosine alone (for the quadratic field) 
observations can be phased together with our 5 older ones within an accuracy of 0.01 cycle. However, when our magnetic measurements are plotted against phase, no systematic trend is seen for any of the studied field moments, even though our observations sample rather well the rotation period. This is not too surprising, given the low level of rotational variability shown by the star in photometry or in spectroscopy. As a consequence, no progress is made in the knowledge of the magnetic field of HD 128898, with respect to the results already reported in Papers II to $\mathrm{V}:\left\langle H_{\mathrm{z}}\right\rangle$ and $\left\langle x H_{\mathrm{z}}\right\rangle$ are below the detection threshold, while our observations are consistent with a fairly constant quadratic field of the order of $7.5 \mathrm{kG}$.

\subsubsection{HD 137509}

HD 137509 has one of the strongest magnetic fields known in an Ap star (Paper V). The distribution in phase of our 9 observations of this star obtained until 1988 was far from ideal (half of the rotation cycle remained unsampled), so that the variation curves of the various field moments were poorly constrained. The 5 new observations reported here improve this situation, and reveal the contribution of a very significant term with twice the rotation frequency in the variations of the longitudinal field and of the crossover (see Fig. 9). The first harmonic is possibly present in the quadratic field curve too, but for this field moment, our data are not quite conclusive yet. A marked double-wave character of the $\left\langle H_{\mathrm{z}}\right\rangle$ variations such as we find in HD 137509 had been observed so far in the stars HD 37776 (Thompson \& Landstreet 1985) and HD 133880 (Landstreet 1990). This indicates that the magnetic field of HD 137509 has an unusually important quadrupolar component.

\subsubsection{HD 137909}

The 4 new observations of the famous cool Ap star HD 137909 that we present here fill only partly the gap in phase coverage of our 11 older data. But, thanks in particular to their better accuracy, they bring a significant improvement in the definition of the variation curves. For all three field moments considered here, these curves show no departure from sinusoids, in contrast with the very anharmonic behaviour of the field modulus (MHLLM). The quadratic field is approximately 1.25 times larger than the latter close to maximum, while the ratio between both goes down to very nearly 1.0 at their minimum. This remarkably small value of $\left(\left\langle H^{2}\right\rangle+\left\langle H_{z}^{2}\right\rangle\right)^{1 / 2} /\langle H\rangle$, which had already been pointed out in Paper $\mathrm{V}$, is unique among the stars that we have studied. It points to a rather unusual structure of the magnetic field. In particular, it indicates that at quadratic field minimum, the magnetic field of HD 137909 must be seen almost purely transversally. This view is supported by the relative phasing of the curves of variation of $\left\langle H_{\mathrm{z}}\right\rangle$ and of $\left(\left\langle H^{2}\right\rangle+\left\langle H_{\mathrm{z}}^{2}\right\rangle\right)^{1 / 2}$ (which are in

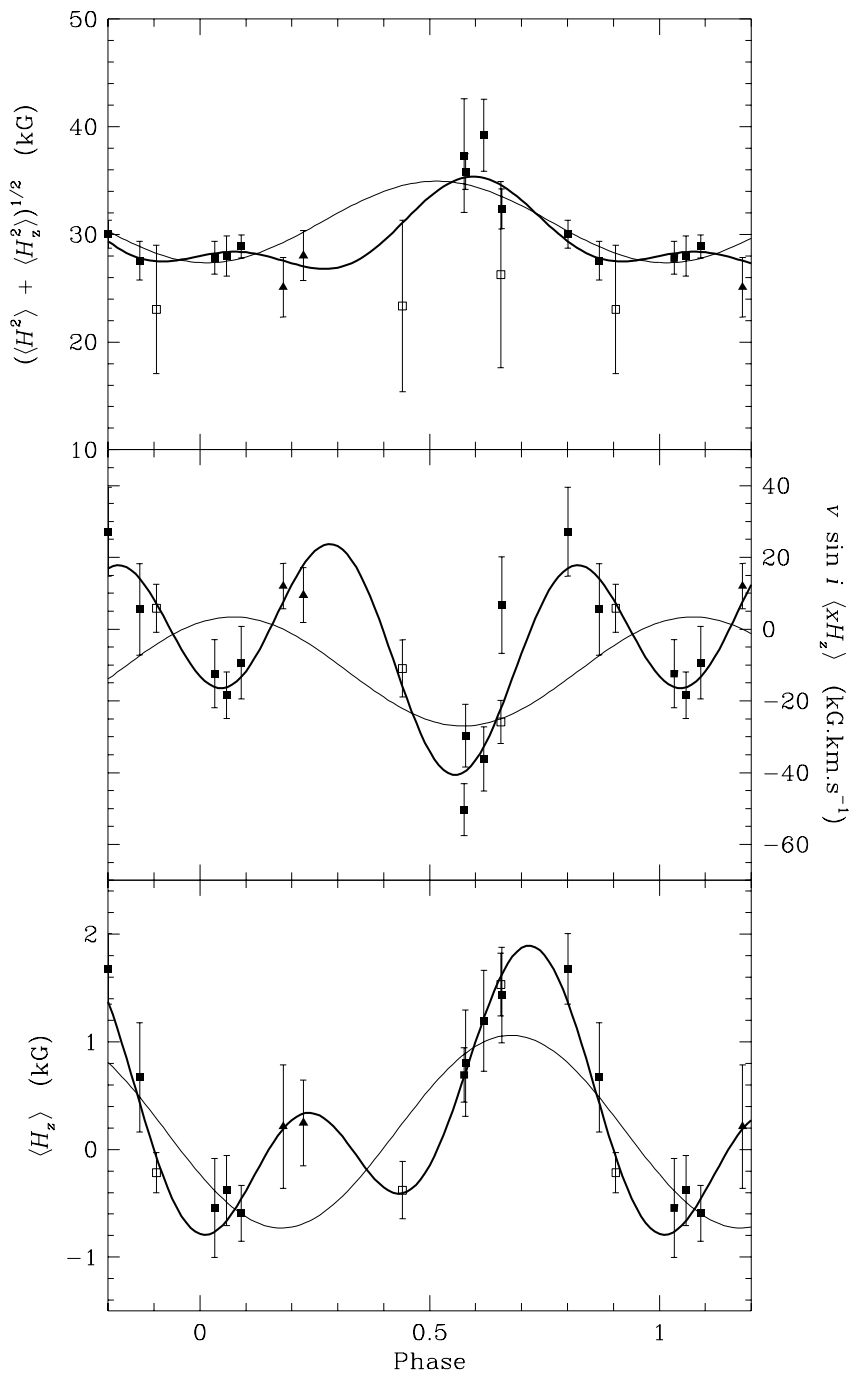

Fig. 9. Phase diagram of our measurements of the mean longitudinal magnetic field (lower panel), of the crossover (centre), and of the mean quadratic magnetic field (upper panel) of HD 137509 (see the text for the meaning of the symbols). The curves are least-squares fits of the data by a cosine wave (thin curves) and by the superposition of a cosine wave and of its first harmonic (thick curves)

quadrature with respect to each other - see Fig. 10 and Tables 4 and 6). But this apparent simplicity is misleading: the actual geometrical structure of the field of this star appears very complex and its detailed modelling is quite challenging (Leroy 1995; Wade 1995).

\subsubsection{HD 147010}

Our 2 new observations of HD 147010 and the derivation of a refined value of its rotation period by Catalano \& Leone (1993) do not significantly change the picture of its magnetic field that we had obtained from our previous 17 observations. Accordingly, the reader is referred to the 


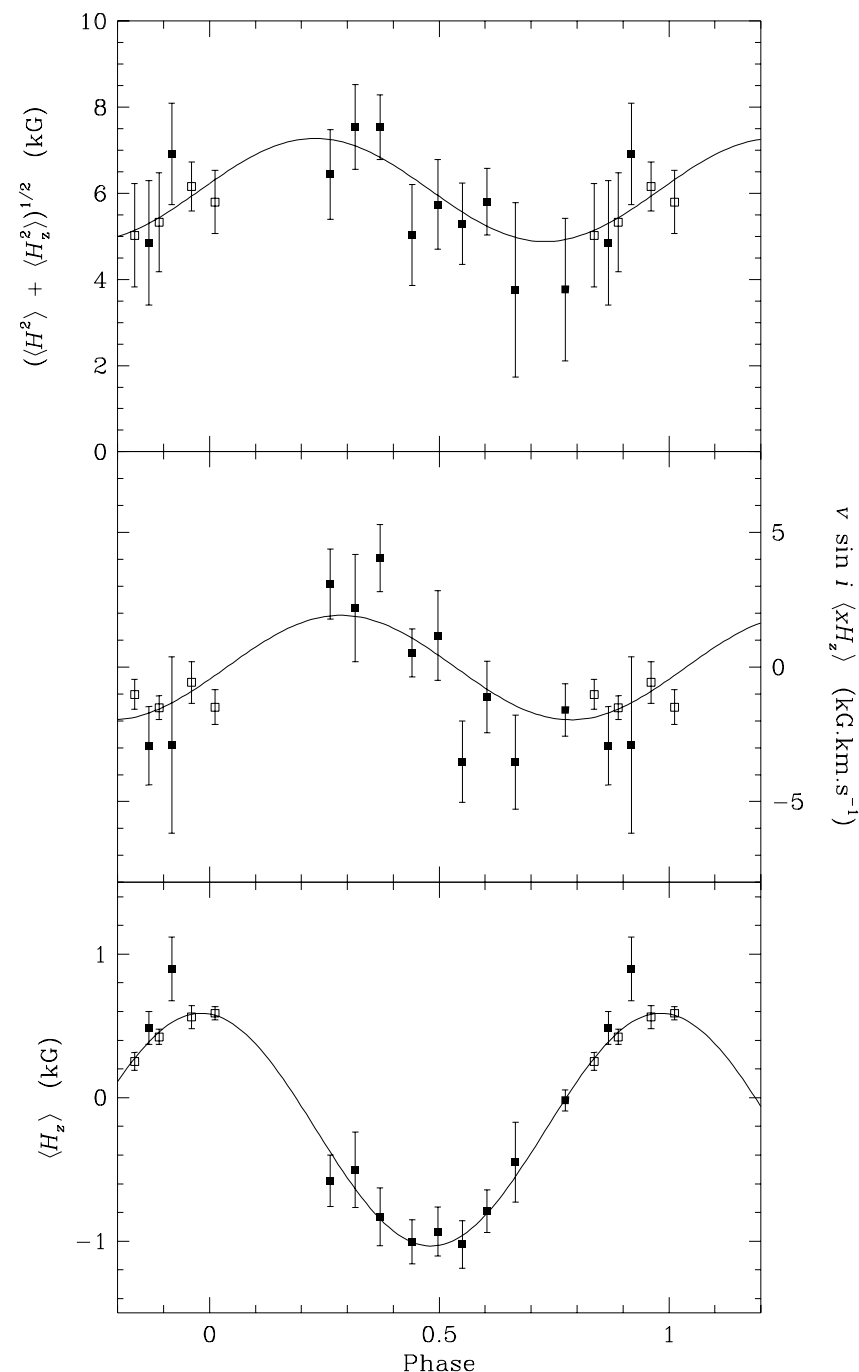

Fig. 10. Phase diagram of our measurements of the mean longitudinal magnetic field (lower panel), of the crossover (centre), and of the mean quadratic magnetic field (upper panel) of HD 137909 (see the text for the meaning of the symbols). The curves are least-squares fits of the data by sinusoids

previous papers of this series for a detailed discussion. The updated variation curves are shown in Fig. 11.

\subsubsection{HD 153882}

Our 14 observations of HD 153882 published so far were not sampling the rotation period ideally, with a gap in the coverage left between phases 0.1 and 0.5 . The 3 new observations reported here all fall in this gap, which allows the variation curves of the magnetic field moments to be better defined. The resulting fits are shown in Fig. 12. While the longitudinal field and the crossover are adequately represented by a sinusoid with the rotation frequency of the star, we fully confirm the suspicion expressed in Paper V

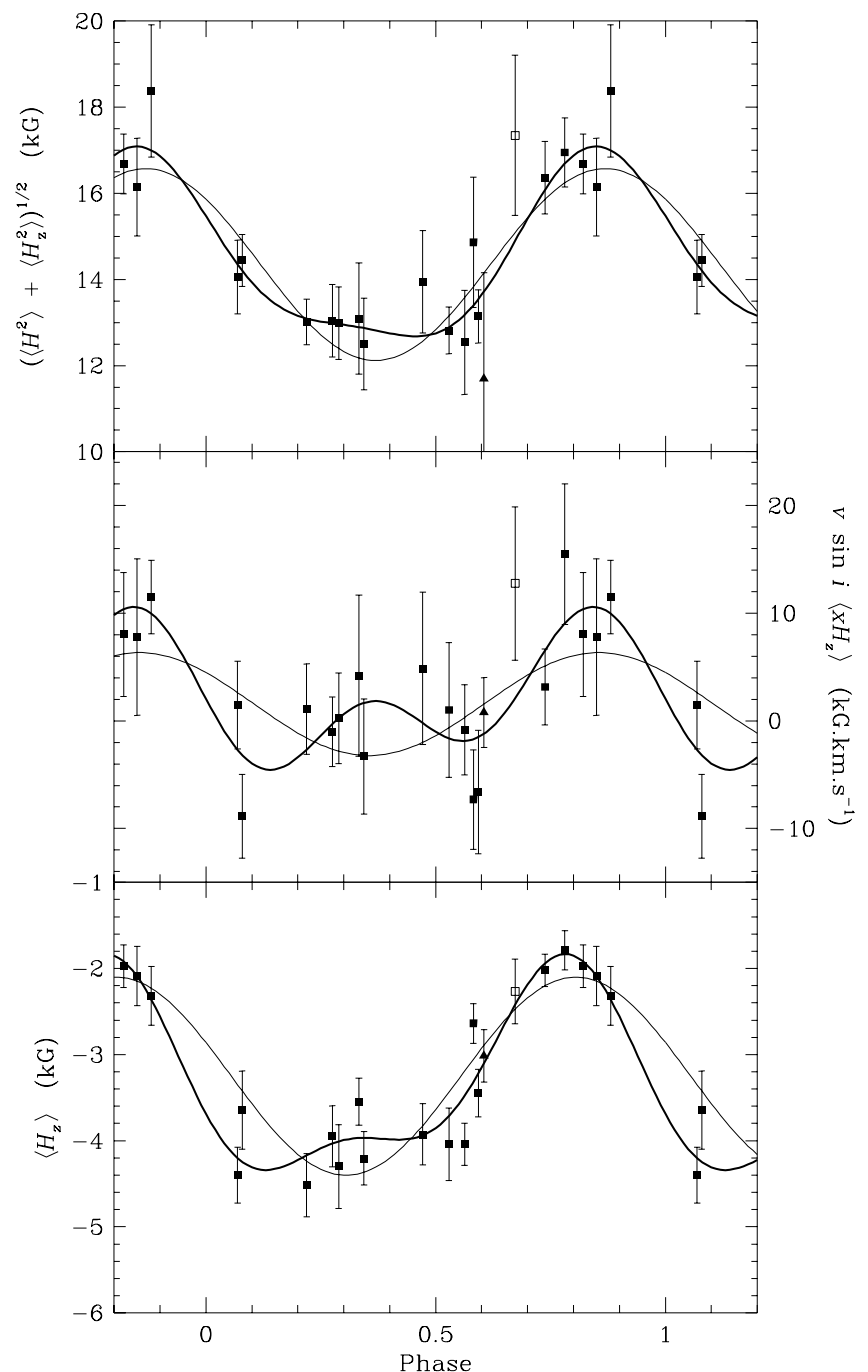

Fig. 11. Phase diagram of our measurements of the mean longitudinal magnetic field (lower panel), of the crossover (centre), and of the mean quadratic magnetic field (upper panel) of HD 147010 (see the text for the meaning of the symbols). The curves are least-squares fits of the data by a cosine wave (thin curves) and by the superposition of a cosine wave and of its first harmonic (thick curves)

that the best fit to the quadratic field variations is given by a sinusoid with twice the rotation frequency, alone.

\subsubsection{HD 165474}

Preston (1971) has discovered resolved magnetically split lines in the spectrum of HD 165474 observed in unpolarized light. The mean magnetic field modulus of this star has been studied by MHLLM. According to these authors, $\langle H\rangle$ appears to undergo low-amplitude (200 to $300 \mathrm{G}$ peak-to-peak) variations about a mean value of $6.5 \mathrm{kG}$, over a (rotation) period which could not be uniquely 


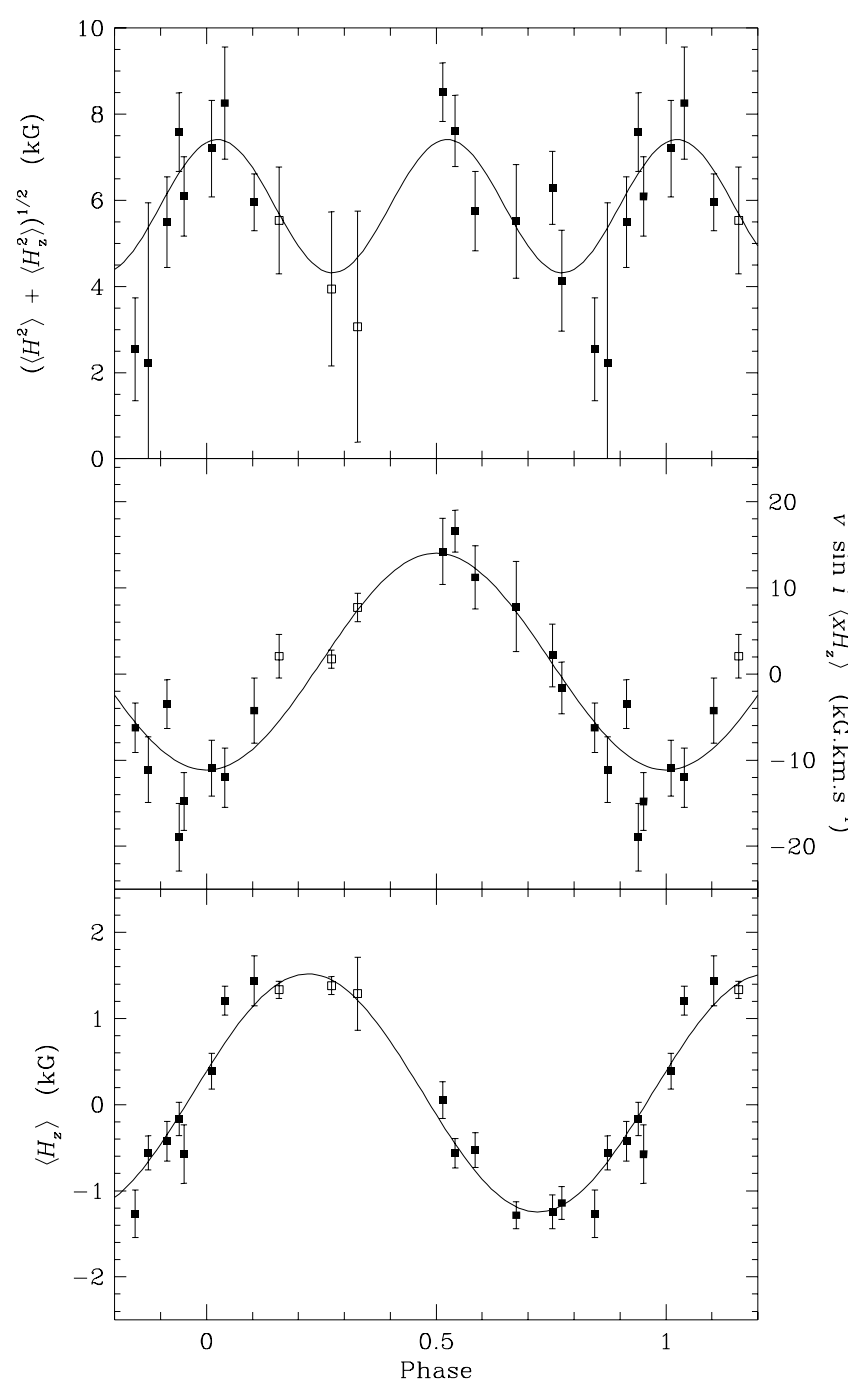

Fig. 12. Phase diagram of our measurements of the mean longitudinal magnetic field (lower panel), of the crossover (centre), and of the mean quadratic magnetic field (upper panel) of HD 153882 (see the text for the meaning of the symbols). The curves are least-squares fits of the data by cosine waves with the rotation frquency of the star (longitudinal field and crossover) or with twice that frequeny (quadratic field)

determined, but which may plausibly be quite short (the most probable value being 2.54 ).

Given the fairly large value of the field modulus, it is surprising that our 3 determinations of the longitudinal field all yield null values. In particular, the 2 new measurements reported here are significantly more accurate than the one published in Paper III and allow us to set a rather conservative $3 \sigma$ upper limit of the order of $210-240 \mathrm{G}$ (in absolute value) for the longitudinal field of HD 165474. However, preliminary visual examination of an additional CASPEC observation of HD 165474 obtained in July 1996 shows the unmistakable signature of a definite longitudi- nal field. Thus our 3 null measurements so far just appear to be very unfortunately phased.

We have not detected any crossover in HD 165474 (with a quite stringent $3 \sigma$ upper limit of $1.5 \mathrm{~km} \mathrm{~s}^{-1} \mathrm{kG}$ in our last observation). This is not quite unexpected for a star having resolved magnetically split lines, but the result is nonetheless non-trivial if the rotation period is as short as 2.54 (note that HD 137909, another star with magnetically resolved lines with a rotation period of 18.5 , does show significant crossover).

Our 2 new determinations of the quadratic field yield values of this field moment of the order of $7.0 \mathrm{kG}$, while a value of $10.1 \mathrm{kG}$ had been obtained in Paper V. However, the uncertainty of the latter was larger, so that there is no major inconsistency between it and the more recent measurements, within the limits of their respective accuracies. Tentatively adopting $7.0 \mathrm{kG}$ as the representative value of the quadratic field, the ratio between this field moment and the mean field modulus (MHLLM) is of the order of 1.08 .

\subsubsection{HD 168733}

From photometric observations, Manfroid \& Renson (1994) have derived an unambiguous value of the rotation period of HD $168733(P=6$ d 3540$)$, which however seemed inconsistent with the longitudinal magnetic field measurements of Jones \& Wolff (1974). The 4 observations of this star reported here were obtained as part an effort to solve this discrepancy. Together with 3 former observations discussed in Papers II to V, they sample well the rotation cycle. Still, the standard deviation of the resulting $7\left\langle H_{\mathrm{z}}\right\rangle$ measurements is only $130 \mathrm{G}$, that is, quite comparable to the typical uncertainty of the individual determinations. Thus, we are not detecting any significant $\left\langle H_{\mathrm{z}}\right\rangle$ variation. We are led to speculate that, as is known to have occurred frequently for $\left\langle H_{\mathrm{z}}\right\rangle$ diagnosis from photographic plates, Jones \& Wolff (1974) somewhat underestimated the uncertainty of their measurements (they quote a typical probable error of $150 \mathrm{G}$ ), and that the standard deviation of their data $(310 \mathrm{G})$ only reflects their random errors. Thus we argue that the longitudinal field of HD 168733 is not detectably variable, and that its mostly constant value is close to the average of our 7 determinations, $-636 \mathrm{G}$ (which is consistent with the average of all the measurements of Jones \& Wolff: $-688 \mathrm{G}$ ).

The absence of a measurable crossover in HD 168733 is not surprising, given that fairly sharp spectral lines had been observed at high dispersion by Mathys \& Lanz (1992). We had been unable to determine the quadratic field in Paper V, and we achieve only one marginally significant detection here $(3.7 \mathrm{kG}$ at the $3.3 \sigma$ level $)$. 
The fit of a cosine to our $\left\langle H_{\mathrm{z}}\right\rangle$ measurements, shown in the lower panel of Fig. 14 (coefficients in Table 4), must also be regarded as provisional, although consideration of Babcock's data (see Fig. 33 of Paper II) suggests that it is probably not too far from reality.

The ratio between the quadratic field and the mean field modulus between phases 0.8 and 0.2 (where $\langle H\rangle$ is fairly constant) is approximately 1.50 .

Of course, the very slow rotation of HD 187474 rules out any possibility of detection of crossover.

\subsubsection{HD 188041}

HD 188041 is another long period (223.9, see Hensberge 1993) star with resolved lines (Preston 1971). Our 7 observations ( 4 of which had already been discussed in the previous papers of this series) are insufficient to characterize the behaviour of its longitudinal field by themselves, but they appear roughly consistent with Babcock's (1954, 1958) data.

No significant crossover nor quadratic field was detected in this star.

\subsubsection{HD 201601}

The slow drift of the longitudinal field of HD 201601 from 1946 to 1988 has been illustrated in Fig. 37 of Paper II. Leroy et al. (1994) have presented compelling arguments (based on broad-band linear polarization measurements) definitely establishing that the observed $\left\langle H_{z}\right\rangle$ variation does result from stellar rotation with an extraordinarily long period exceeding 70 years. Some ambiguity is left on the exact value of this period. According to Leroy et al. (1994), its smallest plausible value appears close to 77 years, but it might possibly be as long as 110 years. For a period close to the lower limit of this range, the negative extremum of $\left\langle H_{\mathrm{z}}\right\rangle$ was expected in 1994, while longer periods allow the longitudinal field to keep becoming more negative for a while still. The 4 new measurements of $\left\langle H_{\mathrm{z}}\right\rangle$ reported here show some hint of a flattening of the variation curve, which may be indicating that the star is indeed approaching its negative extremum and would accordingly suggest that the period is close to the low end of the acceptable range. That the mean field modulus (diagnosed from the observation of magnetically resolved lines) also seems to be reaching its maximum (MHLLM) may be another indication of this but one has to remember that the $\left\langle H_{\mathrm{z}}\right\rangle$ and $\langle H\rangle$ extrema do not necessarily coincide in phase.

Given the long rotation period, one cannot expect to observe crossover in HD 201601.

For the reasons exposed in Sect. 3, quadratic field diagnosis is more difficult and less accurate here than in Paper V. This explains why, for HD 201601, quadratic field could be diagnosed only from the spectra taken with the long camera of CASPEC, and why in spite of the higher dispersion of the latter, the uncertainty affecting those 2 determinations is larger than that of the measurements of Paper V. Accordingly, no real progress is achieved here in the knowledge of this field moment. Based on the (better) data of Paper V alone and on the only field modulus measurement of MHLLM contemporaneous with them, the ratio $\left(\left\langle H^{2}\right\rangle+\left\langle H_{\mathrm{z}}^{2}\right\rangle\right)^{1 / 2} /\langle H\rangle$ is found to be of the order of 1.85 .

\subsection{Additional stars}

\subsubsection{HD 2453}

MHLLM have derived a refined value of $521 \mathrm{~d}$ for the rotation period of HD 2453, an Ap star with resolved magnetically split lines (Mathys \& Lanz 1992). They have shown that with this value of the period, the measurement of $\left\langle H_{\mathrm{z}}\right\rangle$ reported here is consistent with those of Babcock (1958) and of Wolff (1975). The slow rotation of the star does not allow crossover to be observed and the quadratic field cannot be determined.

\subsubsection{HD 5737}

Our single observation of HD 5737 yields null values of $\left\langle H_{z}\right\rangle$ and $\left\langle x H_{z}\right\rangle$, while we could not diagnose the quadratic field. The longitudinal field had already been studied in detail by Shore et al. (1990). Their $16 \mathrm{H} \beta$ photopolarimetric determinations of this field moment, together with 7 older measurements of Borra et al. (1983), revealed that it varies mostly sinusoidally between -0.3 and $+0.5 \mathrm{kG}$ over the stellar rotation period. The most accurate value of the latter, 21. 652 , has been derived by Manfroid \& Renson (1994) from photometric observations in the Strömgren system. Using it, our observation can be phased with respect to those of Shore et al. (1990) with an accuracy of 0.03 rotation cycle. Our $\left\langle H_{z}\right\rangle$ value of $(-322 \pm 140) \mathrm{G}$, derived at phase 0.312 , is quite consistent with the value of $-314 \mathrm{G}$ predicted for that phase from the best fit cosine to the data of Borra et al. (1983) and of Shore et al. (1990).

\subsubsection{HD 6532}

HD 6532 is a roAp star with a short rotation period: 1.94 (see Kurtz et al. 1996 and references therein). As a result of this fast rotation, its spectral lines show significant Doppler broadening, which complicates the magnetic field diagnosis. Our observation is, to our knowledge, the first attempt to detect the magnetic field of this star. While it yielded null values of the longitudinal field and of the crossover, a quadratic field of about $22 \mathrm{kG}$ was measured. That $\left\langle H_{\mathrm{z}}\right\rangle$ and $\left\langle x H_{\mathrm{z}}\right\rangle$, which generally vary in phase quadrature, are both small at a given phase suggests that the longitudinal field never gets very large. Although it remains to be confirmed by observations at other phases, we note that this behaviour is similar to that of the only 
two other fast rotating roAp stars whose magnetic field has been studied so far. Indeed, these stars, HD 83368 and HD 128898, also have relatively modest longitudinal fields and quite strong quadratic fields (see Sects. 4.2.2 and 4.2.8).

\subsubsection{HD 19918}

HD 19918 is another roAp star (Martinez \& Kurtz 1994) whose magnetic field has never been studied. Here we report the detection of a longitudinal field at the $3.8 \sigma$ level. Null values are derived for both the crossover and the quadratic field. But the constraint on the latter is very weak, with a $3 \sigma$ upper limit of $21 \mathrm{kG}$. A high-resolution $(\lambda / \Delta \lambda \approx 115000)$ observation in unpolarized light, performed with the ESO Coudé Echelle Spectrograph (CES) fed by the $3.6 \mathrm{~m}$ telescope is significantly more stringent. Indeed, from this spectrum, the lines of HD 19918 appear quite sharp, indicating that the field modulus of this star is unlikely to exceed much $1 \mathrm{kG}$.

\subsubsection{HD 22920}

Borra et al. (1983) concluded that HD 22920 almost certainly has a weak longitudinal field, from the consideration of the average of four measurements performed by $\mathrm{H} \beta$ photopolarimetry, none of which taken alone yields a firm detection. Our single attempt to determine $\left\langle H_{z}\right\rangle$ is not more successful. But it is noteworthy that it gives a small positive $\left\langle H_{\mathrm{z}}\right\rangle$ value quite consistent with the data of Borra et al. (1983), thus that it supports these authors' argument about the reality of the field. We detect no crossover in HD 22920 and we cannot diagnose its quadratic field.

\subsubsection{HD 36485}

6 determinations of the longitudinal field of HD 36485 have been performed through $\mathrm{H} \beta$ photopolarimetry by Bohlender et al. (1987), and these authors have concluded that $\left\langle H_{\mathrm{z}}\right\rangle$ in this star is constant, at a value of $-3.4 \mathrm{kG}$. However, our single $\left\langle H_{z}\right\rangle$ determination yields a value of $-1.9 \mathrm{kG}$. We cannot decide at present whether the discrepancy with the conclusion of Bohlender et al. (1987) means that these authors have misinterpreted their data or if it results from the use of different measurement techniques. Indeed, inconsistencies between longitudinal fields diagnosed with the Zeeman analyzer of CASPEC and with the $\mathrm{H} \beta$ photopolarimeter have been found for several stars and are not fully understood (see Paper II; see also Mathys 1989). But they seldom are so extreme as the difference obtained here for HD 36485.

No significant crossover is detected in HD 36485, and its quadratic field cannot be diagnosed.

\subsubsection{HD 37058}

Sargent et al. (1967) performed the first determination of the longitudinal field of HD 37058 and derived a value of $(2500 \pm 450) \mathrm{G}$. Conti (1970) obtained 5 measurements ranging from +30 to $+1300 \mathrm{G}$, with uncertainties of 400-600 G. None of Borra et al.'s (1983) 3 individual values of $\left\langle H_{\mathrm{z}}\right\rangle$ (all negative) quite reach the $3 \sigma$ level (the $\sigma$ of each of the 3 measurements is just slightly larger than $300 \mathrm{G}$ ), but their average indicates that a field is detected at the $99.9 \%$ confidence level. Therefore Borra et al. (1983) argue that all observations are consistent with a reversing longitudinal field varying with a peak-to-peak amplitude of 2 to $3 \mathrm{kG}$. If this interpretation is correct, our determination of $\left\langle H_{\mathrm{z}}\right\rangle$, which is much more accurate than the previous ones, must have been very unfortunately phased, since it yields a null result too. The value of the rotation period derived by Pedersen (1979) is regrettably too coarse to test the relative phasing of our observation with respect to older ones. But the reality of the field of HD 37058 is supported by our finding of a fairly significant crossover (at the $4.1 \sigma$ level). We also measure a marginally significant quadratic field, at the $2.7 \sigma$ level.

\subsubsection{HD 50169}

The measurements of the mean magnetic field modulus of HD 50169 by MHLLM indicate that the period of rotation of this star with magnetically resolved lines, discovered by Mathys \& Lanz (1992), must be much longer than 4 years. Consistently with this, Babcock (1958) observed a slow increase of $\left\langle H_{\mathrm{z}}\right\rangle$ from $+670 \mathrm{G}$ in 1953 to $+2120 \mathrm{G}$ three years later. Our own measurement falls between these two values.

Comparison of the value of $\left(\left\langle H^{2}\right\rangle+\left\langle H_{\mathrm{z}}^{2}\right\rangle\right)^{1 / 2}$ found here with contemporaneous $\langle H\rangle$ data of MHLLM indicate that the ratio between the two quantities is of the order of 1.7. Of course, no crossover is detected.

\subsubsection{HD 55719}

HD 55719 is one of the three double-lined spectroscopic binaries definitely known to contain a magnetic Ap star. The latter has resolved magnetically split spectral lines (Mathys 1990). Both the characteristics of the binary system and the longitudinal field of the Ap component have been studied in detail by Bonsack (1976). This author's $\left\langle H_{\mathrm{z}}\right\rangle$ measurements and his determination of the rotation period have been rediscussed in detail by MHLLM. Indeed, none of the two values of the period that he favours is consistent with the field modulus measurements. MHLLM could not definitely establish the value of the rotation period, but 847 or 775 days appear as most plausible. Nevertheless, it seems unavoidable that 2 or 3 of Bonsack's (1976) measurements are bound to be inconsistent with the bulk of his data, regardless of what the rotation period is (they were already discrepant with 
Bonsack's preferred period values). Except for these discrepant values, all of Bonsack's (1976) $\left\langle H_{\mathrm{z}}\right\rangle$ data are larger than our 3 measurements. If the interpretation proposed by MHLLM that the rotation period is longer than 2 years is correct, this hints at the existence of systematic differences betwen Bonsack's (1976) and our longitudinal field determinations. This is not unusual but it implies that we need to accumulate more data before we can hope to use the $\left\langle H_{\mathrm{z}}\right\rangle$ measurements to constrain the rotation period.

The crossover measured in our third observation is too marginal (at the $3.3 \sigma$ level) to question the long period hypothesis. The first two quadratic field determinations (based on spectra recorded with the long camera of CASPEC) are significantly more accurate than the third one (for which the short camera was used). Their ratio to the average field modulus of HD 55719 (MHLLM) is close to 1.3 .

\subsubsection{HD 70331}

The rotation period of HD 70331, one of the hottest Ap stars with magnetically resolved lines, could not be uniquely determined from the consideration of its mean magnetic field modulus, but it must probably be short, two of the most plausible values being 3.03 and 3.65 (MHLLM). Longitudinal field measurements may prove very useful to establish the value of the period more reliably. This field moment is determined here for the first time, yielding a large negative value of $-2.8 \mathrm{kG}$. As is often the case for stars with magnetically resolved lines, no significant crossover is detected (although one cannot exclude to observe it at other phases). The large quadratic field is about 1.1 to 1.2 times larger than the field modulus.

\subsubsection{HD 81009}

Our single determination of the longitudinal field of HD 81009 is fully consistent with an unpublished variation curve of this moment, obtained by G. Hill and D. Bohlender (Hill, private communication; see also MHLLM). Rather remarkably, given the rather long rotation period of 33.96 (Waelkens 1985), crossover is detected at a fairly significant level $(3.8 \sigma)$. This is fully consistent with the observation of some Doppler distortion in the split components of the magnetically resolved lines of this star (resolved magnetically split lines have first been observed in this star by Preston 1971). The ratio $\left(\left\langle H^{2}\right\rangle+\left\langle H_{\mathrm{z}}^{2}\right\rangle\right)^{1 / 2} /\langle H\rangle$ at the phase of our observation is approximately 1.75 .

\subsubsection{HD 93507}

HD 93507 is another star with magnetically resolved lines discovered by MHLLM, whose longitudinal field has never been measured before. The two determinations presented here are separated by 0.302 rotation cycle (the period is
$556 \mathrm{~d})$. That they differ by $1 \mathrm{kG}$ suggests that $\left\langle H_{\mathrm{z}}\right\rangle$ undergoes quite sizeable variations. The second determination of the quadratic field, based on a spectrum taken with the long camera, is considerably more accurate than the first one, for which the short camera of CASPEC was used. We only compare the former to the field modulus at the same phase: the ratio between them is 1.07. HD 93507 rotates too slowly to show observable crossover.

\subsubsection{HD 116114}

The observation of magnetically resolved lines in HD 116114 has first been reported by Mathys et al. (1993). From the mean field modulus measurements, it was inferred that the rotation period must be much longer than 3 years (MHLLM). The first measurement of $\left\langle H_{\mathrm{z}}\right\rangle$ which we report here yields a large negative value $(-1.9 \mathrm{kG})$, while the quadratic field is 1.2 times larger than the field modulus. No crossover is detected.

\subsubsection{HD 134214}

The roAp star HD 134214 (Kreidl 1985) has one of the smallest field moduli measured by MHLLM in any star with resolved magnetically split lines. These authors were unable to derive a definite value of the period, but the latter may well be short. A tentative value of 4.1456 is suggested by MHLLM. Should this value prove correct, the two observations reported here would be separated by only 0.104 rotation cycle. The fact that no significant measurement of either $\left\langle H_{\mathrm{z}}\right\rangle$, or $\left\langle x H_{\mathrm{z}}\right\rangle$, or $\left(\left\langle H^{2}\right\rangle+\left\langle H_{\mathrm{z}}^{2}\right\rangle\right)^{1 / 2}$ is obtained from any of them would then only be a weak constraint.

\subsubsection{HD 137949}

MHLLM have shown that all previous measurements of $\left\langle H_{\mathrm{z}}\right\rangle$ (Babcock 1958; van den Heuvel 1971; Wolff 1975) in the roAp star HD 137949 (Kurtz 1982), together with our two measurements presented here, consistently indicate that the star has a very long rotation period (possibly more than 75 years). The crossover measured from our first observation is not significant enough (at the $3.3 \sigma$ level) to challenge this conclusion. Our two spectra were taken with the short camera of CASPEC, and the $\left(\left\langle H^{2}\right\rangle+\right.$ $\left.\left\langle H_{\mathrm{z}}^{2}\right\rangle\right)^{1 / 2}$ determinations have uncertainties too large to be really meaningful.

\subsubsection{HD 144897}

We are reporting the first determination of the longitudinal field of HD 144897, an Ap star with resolved magnetically split lines (MHLLM). The phase of this observation is about mid-way between the maximum and the minimum of the field modulus. If the $\left\langle H_{\mathrm{z}}\right\rangle$ and $\langle H\rangle$ extrema roughly coincide in phase (which is not infrequent, even though some stars show large departures from this phase 
relation), we expect $\left\langle H_{z}\right\rangle$ to reach at its maximum a value significantly greater than the already large $+2.0 \mathrm{kG}$ derived here. Not surprisingly, since the rotation period is fairly long (48.43), we observe no crossover. Comparing our good measurement of $\left(\left\langle H^{2}\right\rangle+\left\langle H_{\mathrm{z}}^{2}\right\rangle\right)^{1 / 2}$ with $\langle H\rangle$ at the same phase, the ratio of the two is found to be 1.13 .

\subsubsection{HDE 318107}

Magnetically resolved split lines had been sought (and found - see MHLLM) in HDE 318107 following North's (1987) report that the rotation period of this star is 52 d. Somewhat ironically, the field modulus measurements proved inconsistent with this value of the period. They were however too noisy to allow MHLLM to establish what the actual value of the period is. Longitudinal field measurements may prove most useful to this effect, since the variations of this field moment are often (relatively) larger than those of $\langle H\rangle$. The first determination of $\left\langle H_{\mathrm{z}}\right\rangle$ reported here is promising, since a large positive value (almost $2.0 \mathrm{kG}$ ) is obtained, with a reasonably small uncertainty $(230 \mathrm{G})$. The achieved determination of the quadratic field is pretty accurate too. The ratio of $\left(\left\langle H^{2}\right\rangle+\left\langle H_{\mathrm{z}}^{2}\right\rangle\right)^{1 / 2}$ to the average of the $\langle H\rangle$ measurements of MHLLM is close to 1.45 .

\subsubsection{HD 166473}

Our interest in HD 166473, originally motivated by the fact that it is a roAp star (Kurtz \& Martinez 1987), grew when resolved magnetically split lines were discovered in its spectrum (MHLLM). The mean magnetic field modulus of this star varies slowly, with a period still unknown, but definitely much longer than 3.2 years, and a large relative amplitude. Our 3 measurements of $\left\langle H_{z}\right\rangle$ (the first ones ever obtained for this star) have been performed over 2 months, close to the maximum of $\langle H\rangle$. Not surprisingly, $\left\langle H_{\mathrm{z}}\right\rangle$ shows no significant variation over that time interval. No crossover is detected. From the last two, more accurate (long camera) determinations of the quadratic field, the ratio of the latter to the field modulus at the time of our observations was 1.30 .

\subsubsection{HD 176232}

The first study of the longitudinal field of HD 176232, a roAp star (Heller \& Kramer 1988), was performed by Babcock (1958). If the uncertainties he quotes for his 6 determinations of $\left\langle H_{z}\right\rangle$ are not underestimated (as has unfortunately often been the case for photographic $\left\langle H_{z}\right\rangle$ measurements), these determinations rank among the most accurate ones ever achieved, and all of them indicate that HD 176232 has a small (between -315 to $+440 \mathrm{G}$ ) but definite longitudinal field. The accuracy of our measurement of $\left\langle H_{z}\right\rangle$ is significantly worse than the accuracy claimed by Babock (1958), and we do not detect this field moment, nor crossover or quadratic field.

\subsubsection{HD 193756}

We neither detect any of the three field moments considered in this study in the roAp star HD 193756 (Martinez $\&$ Kurtz 1990). We are not aware of any other attempt to diagnose the magnetic field of this star.

\subsubsection{HDE 335238}

HDE 335238 has long been known to have magnetically resolved spectral lines, from which a large, variable field modulus is derived (Preston 1971). But probably because of its faintness, it has not been paid much attention until MHLLM's recent systematic study of the magnetic field modulus. These authors were unable to derive an unambiguous value of the rotation period, but they showed that it must without doubt be between 40 and 50 days. The phase of our observation is accordingly unknown, and in particular, we cannot relate the quadratic field that we determine $(+10.5 \mathrm{kG})$ to the field modulus. We also detect a fairly large negative longitudinal field, and more unexpectedly, a (somewhat marginal) crossover (at the $3.4 \sigma$ level).

\subsubsection{HD 203932}

HD 203932 is another roAp star (Kurtz 1984) whose magnetic field has never been studied before. Our two attempts yield no detection of the longitudinal field or of the crossover, and we were unable to diagnose the quadratic field.

\subsubsection{HD 216018}

We find no significant variation of $\left\langle H_{\mathrm{z}}\right\rangle$ nor of $\left(\left\langle H^{2}\right\rangle+\right.$ $\left.\left\langle H_{\mathrm{z}}^{2}\right\rangle\right)^{1 / 2}$ between our 3 observations (spread over slightly less than one year) of HD 216018. We do not detect any crossover either. All this is consistent with the very long rotation period (much longer than 3 years) inferred from the variations of the mean field modulus of this Ap star with magnetically resolved lines (MHLLM). The ratio of the quadratic field to the field modulus is of the order of 1.25 .

\subsubsection{HD 217522}

The only hint of a magnetic field that we find in the roAp star HD 217522 (Kurtz 1983) is provided by a very marginal $(2.4 \sigma)$ quadratic field measurement. Our single observation (which is the first one aimed at detecting a field in this star) does not show any significant longitudinal field or crossover.

\subsubsection{HD 218495}

HD 218495 is another roAp star (Martinez \& Kurtz 1990) whose magnetic field has never been studied. That both the $\left\langle H_{\mathrm{z}}\right\rangle$ and $\left\langle x H_{\mathrm{z}}\right\rangle$ determinations yield nonzero values 
at a low level of significance ( $2.2 \sigma$ and $2.3 \sigma$, resp.) may be purely coincidental, but it may also indicate that the star indeed has measurable longitudinal field and crossover and that our single observation was unfortunately phased approximately mid-way between the extrema of both those field moments. The quadratic field could not be diagnosed.

\section{Conclusion}

We have presented 95 new determinations of the mean longitudinal magnetic field and of the crossover in 44 Ap stars, which complement those already published in Papers III and IV. For the mean quadratic magnetic field, only 79 measurements are reported: the attempt to diagnose this field moment yielded a result devoid of physical meaning (a negative value of the mean square magnetic field) for 16 observations. As already noted in Sect. 3, the quadratic field diagnosis proved more difficult than in Paper V, since the changes of instrumental configuration between the various observations of a given star prevented us from combining them to derive a single contribution for the combination of the non-magnetic part of the line profile and of the instrumental profile. The reduced wavelength coverage achieved with the long camera of CASPEC used with a "small" $(512 \times 512$ pixels $)$ CCD, and the resulting small number of lines available in some cases to diagnose the field, also severely hampered our ability to derive a meaningful quadratic field.

As indicated in Sect. 1, one of the purposes of the observations reported here was to monitor the evolution of the performance of the Zeeman analyzer of CASPEC throughout the modifications progressively undergone by the instrument. To this effect, we reobserved several of the stars that had already been studied in detail in our previous work. The present determinations of the longitudinal field and of the crossover for these stars (e.g., HD 125248, HD 137909, HD 147010, ...) are consistent with those of Papers III and IV, indicating that the polarimetric performance of CASPEC has remained mostly unaltered through the configuration changes. With the long camera of CASPEC, the uncertainty of the best measurements of $\left\langle H_{\mathrm{z}}\right\rangle$ (achieved for stars with rich, sharplined spectra such as HD 137909 and HD 201601) has gone down to $45-55 \mathrm{G}$. This improvement with respect to the smallest errors of $80-90 \mathrm{G}$ obtained in Paper III reflects well the increase of resolving power by a factor of about 2 resulting from the change from short to long camera. Similar progress is achieved in the accuracy of the crossover determinations (again, see e.g. HD 137909), where the smallest errors are in the $400-500 \mathrm{~km} \mathrm{~s}^{-1} \mathrm{G}$ range. By contrast, the accuracy of quadratic field data is often worse than, and at best similar to that achieved in Paper V, due to the already repeatedly stressed impossibility to combine observations obtained at different phases for the derivation of this field moment. As mentioned in the previous papers of this series, the formal errors that we determine from the rms of the least-squares fits performed to derive the field moments appear as good estimates of the actual measurement uncertainties. This is supported, in particular, by the fact that, for stars repeatedly observed throughout their rotation cycle, a reduced $\chi^{2}$ close to 1 is generally obtained when fitting the data by functions of the types given by Eqs. (4) or (5). This indicates that the uncertainties assigned to the measurements are consistent with their scatter about a smooth variation curve.

For several of the stars, the new measurements obtained here allowed significant improvement to be achieved in the definition of the variation curves. This happens, in particular, for HD 137509 (see Sect. 4.2.10), whose magnetic field is as a result shown to be predominantly quadrupolar, a structure which had been observed so far in only a couple of stars (Thompson \& Landstreet 1985; Landstreet 1990). HD 137509 had already been pointed out in Paper V as a star of particular interest, for having the strongest quadratic field measured so far. The present finding of a strong quadrupolar component in its magnetic field still increases this interest.

Our new quadratic field data for HD 153882 bring full confirmation of the suspicion expressed in Paper $\mathrm{V}$ that the variation of that field moment is best represented by a sinusoid with twice the stellar rotation frequency, with no significant contribution of a term with the rotation frequency. This does not imply any peculiar structure of the magnetic field: depending on the geometry of the observation, such behaviour of $\left(\left\langle H^{2}\right\rangle+\left\langle H_{\mathrm{z}}^{2}\right\rangle\right)^{1 / 2}$ might be seen for a centred dipole (very much like in the case of the mean field modulus, which has been discussed in some detail by MHLLM).

The knowledge of the rotation period of several of the studied stars was improved as a result of the measurements reported here. A refined value of the period of HD 119419 has been derived, thanks to the longer timebase now covered by the avaible data. New measurements of the longitudinal field of HD 24712 almost certainly solve the ambiguity between the values of the period proposed by Kurtz \& Marang (1987) and in Paper II, providing strong evidence that the latter is indeed the correct one. As shown already by MHLLM, combining our $\left\langle H_{z}\right\rangle$ determinations with those of other authors for HD 137949 gives clear indications that the rotation period of that star must be at least of the order of 75 years, making it one of the two Ap stars with the longest period currently known.

In the course of this study, 14 roAp stars (out of 28 such stars currently known) have been observed. For 6 of them, no attempt to detect a magnetic field had ever been made. Magnetic fields are believed to play an essential rôle in roAp stars. According to the most popular model for those stars, the oblique pulsator model (Kurtz 1982), the pulsation modes are aligned with the magnetic axis of the star. Even more fundamentally, most assumptions made about the excitation mechanism of the pulsation 
require the presence of a magnetic field. This stresses the interest of studies of magnetic fields in roAp stars: even the most basic question whether all those stars do have a magnetic field is crucial. The following elements of answer are brought by the present study:

- the first measurements of the longitudinal and quadratic fields of HD 166473 have been obtained. It is no surprise that these field moments are large, since the field modulus of this star, diagnosed by MHLLM, is also large (and slowly varying);

- a somewhat marginal determination of the longitudinal field of HD 19918 was achieved;

- no significant longitudinal field was found in HD 6532, but the strong quadratic field of this star appears quite definite. This is similar to HD 128898, already studied in Papers II to V, in which our new observations still fail to yield a definite longitudinal field measurement but confirm the previously diagnosed quadratic field;

- our two attempts to measure $\left\langle H_{z}\right\rangle$ in HD 134214 yielded null results, but the presence of a relatively weak field in the star had been definitely established from the observation of magnetically resolved lines (MHLLM);

- no non-null value of the considered field moments could be measured in HD 193756, HD 203932, HD 217522, and HD 218495 (all stars whose magnetic field had never been studied before);

- our attempts to detect a field in HD 176232 also failed. In this star, the only evidence for the presence of a weak magnetic field rests on photographic longitudinal field measurements (Babcock 1958), whose unusually small estimated uncertainty is probably not beyond question;

- finally, new data have been obtained improving our knowledge of the fields of HD 24712, HD 83368, HD 137949, and HD 201601.

In summary, more investigations of the magnetic field of the roAp stars would be most desirable, since at present, it is not even clear whether some of them are magnetic at all. We are planning to pursue such a study.

The sample considered here also contains 21 Ap stars with resolved magnetically split spectral lines, out of the 42 such stars currently known (MHLLM). For 8 of them, we report the first determination of the longitudinal field. Our two attempts to measure the longitudinal field of HD 134214 were the first ever made; that both of them yielded null results may possibly be purely coincidental and result from an unfortunate phasing of the observations (see Sect. 4.3.14), as it definitely is for HD 165474 (see Sect. 4.2.14). A long-term programme of systematic study of the longitudinal field of the Ap stars with magnetically resolved lines is in progress, with a view to modelling the field structure from the simultaneous consideration of longitudinal field and field modulus (from MHLLM) data well distributed throughout the stellar rotation cycles.
Generally, the results presented here are intended for future use in determinations of the geometric structure of the magnetic fields of the studied stars. This will be the subject of future works.

Acknowledgements. This research has made use of the Simbad database, operated at CDS, Strasbourg, France.

\section{References}

Babcock H.W., 1951, ApJ 114, 1

Babcock H.W., 1954, ApJ 120, 66

Babcock H.W., 1958, ApJS 3, 141

Babcock H.W., 1960, Stellar Magnetic Fields. In: Stellar Atmospheres, Greenstein J. (ed.). University of Chicago Press, Chicago, p. 282

Bohlender D.A., Brown D.N., Landstreet J.D., Thompson I.B., 1987, ApJ 323, 325

Bohlender D.A., Landstreet J.D., Thompson I.B., 1993, A\&A 269, 355

Bonsack W.K., 1976, ApJ 209, 160

Borra E.F., Landstreet J.D., 1975, PASP 87, 961

Borra E.F., Landstreet J.D., Thompson I., 1983, ApJS 53, 151

Catalano F.A., Leone F., 1993, A\&AS 100, 319

Catalano F.A., Renson P., Leone F., 1991, A\&AS 87, 59

Catalano F.A., Kroll R., Leone F., 1992, A\&A 263, 203

Conti P.S., 1970, ApJ 159, 723

Didelon P., 1987, The Messenger 49, 5

Heller C.H., Kramer K.S., 1988, Inf. Bull. Var. Stars 3247

Hensberge H., 1993, Long term variability in CP stars. In: Peculiar versus normal phenomena in A-type and related stars, IAU Coll. No. 138, Dworetsky M.M., Castelli F., Faraggiana R. (eds.). Astron. Soc. Pacific Conf. Ser. 44, 547

Jones T.J., Wolff S.C., 1974, PASP 86, 67

Kreidl T.J., 1985, Inf. Bull. Var. Stars 2739

Kurtz D.W., 1982, MNRAS 200, 807

Kurtz D.W., 1983, MNRAS 205, 3

Kurtz D.W., 1984, MNRAS 209, 841

Kurtz D.W., 1989, MNRAS 238, 261

Kurtz D.W., Marang F., 1987, MNRAS 229, 285

Kurtz D.W., Martinez P., 1987, MNRAS 226, 187

Kurtz D.W., Kanaan A., Martinez P., Tripe P., 1992, MNRAS 255,289

Kurtz D.W., Sullivan D.J., Martinez P., Tripe P., 1994, MNRAS 270, 674

Kurtz D.W., Marang F., van Wyk F., Roberts G., 1996, MNRAS 280, 1

Landstreet J.D., 1990, ApJ 352, L5

Lanz T., Mathys G., 1991, A\&AS 90, 365

Leroy J.L., 1995, A complete magnetic model of beta Corona Borealis. In: La polarimétrie, outil pour l'étude de l'activité magnétique solaire et stellaire, Mein N., Sahal S. (eds.). Observatoire de Paris-Meudon (in press)

Leroy J.L., Bagnulo S., Landolfi M., Landi Degl'Innocenti E., 1994, A\&A 284, 174

Manfroid J., Renson P., 1994, A\&A 281, 73

Martinez P., Kurtz D.W., 1990, Inf. Bull. Var. Stars 3509

Martinez P., Kurtz D.W., 1994, MNRAS 271, 118

Mathys G., 1988, A\&A 189, 179

Mathys G., 1989, Fundam. Cosmic Phys. 13, 143 
Mathys G., 1990, A\&A 232, 151

Mathys G., 1991, A\&AS 89, 121 (Paper II)

Mathys G., 1993, Magnetic Field Diagnosis through Spectropolarimetry. In: Dworetsky M.M., Castelli F., Faraggiana R. (eds.), Proc. IAU Coll. 138, Peculiar versus Normal Phenomena in A-Type and Related Stars. Astron. Soc. Pacific Conf. Ser. 44, 232

Mathys G., 1994, A\&AS 108, 547 (Paper III)

Mathys G., 1995a, A\&A 293, 733 (Paper IV)

Mathys G., 1995b, A\&A 293, 746 (Paper V)

Mathys G., Lanz T., 1992, A\&A 256, 169

Mathys G., Lanz T., 1996 (in preparation)

Mathys G., Stenflo J.O., 1986, A\&A 168, 184 (Paper I)

Mathys G., Stenflo J.O., 1987a, A\&A 171, 368

Mathys G., Stenflo J.O., 1987b, A\&AS 67, 557

Mathys G., Landstreet J.D., Lanz T., 1993, Observations of magnetically split lines in Ap stars. In: Peculiar versus normal phenomena in A-type and related stars, IAU Coll. No. 138, Dworetsky M.M., Castelli F., Faraggiana R. (eds.). Astron. Soc. Pacific Conf. Ser. 44, 300

Mathys G., Hubrig S., Landstreet J.D., Lanz T., Manfroid
J., 1996, A\&AS (in press) (= ESO preprint No. 1194; MHLLM)

North P., 1987, A\&AS 69, 371

Pasquini L., 1993, CASPEC - Update to the Operating Manual, ESO

Pedersen H., 1979, A\&AS 35, 313

Preston G.W., 1970, ApJ 160, 1059

Preston G.W., 1971, ApJ 164, 309

Preston G.W., 1972, ApJ 175, 465

Renson P., Gerbaldi M., Catalano F.A., 1991, A\&AS 89, 429

Sargent W.L.W., Sargent A.I., Strittmatter P.A., 1967, ApJ 147, 1185

Shore S.N., Brown D.N., Sonnenborn G., Landstreet J.D., Bohlender D.A., 1990, ApJ 348, 242

Thompson I.B., Landstreet J.D., 1985, ApJ 289, L9

van den Heuvel E.P.J., 1971, A\&A 11, 461

Wade G.A., 1995, On the magnetic field of $\beta$ Coronae Borealis (preprint)

Waelkens C., 1985, A\&AS 61, 127

Wolff S.C., 1969, ApJ 158, 1231

Wolff S.C., 1975, ApJ 202, 127 